\title{
Elevated mRNA Level of Y-Box Binding Protein I Indicates Unfavorable Prognosis Correlated with Macrophage Infiltration and T Cell Exhaustion in Luminal Breast Cancer
}

\author{
Zhenhuan Lv' \\ Chunli Xue ${ }^{2}$ \\ Lei Zhang ${ }^{3}$ \\ Jujie Sun ${ }^{4}$ \\ Cong Bo (iD) ${ }^{4}$ \\ 'Department of Clinical Pharmacy, \\ Shandong Cancer Hospital and Institute, \\ Shandong First Medical University and \\ Shandong Academy of Medical Sciences, \\ Jinan, People's Republic of China; \\ ${ }^{2}$ Department of Radiotherapy, Yantai \\ Affiliated Hospital of Binzhou Medical \\ University, Yantai, People's Republic of \\ China; ${ }^{3}$ Physical Examination Center, the \\ Affiliated Hospital of Jining Medical \\ College, Jining, People's Republic of \\ China; ${ }^{4}$ Department of Radiation \\ Oncology, Shandong Cancer Hospital and \\ Institute, Shandong First Medical \\ University and Shandong Academy of \\ Medical Sciences, Jinan, People's Republic \\ of China
}

Correspondence: Cong Bo Department of Radiation Oncology, Shandong Cancer Hospital and Institute, Shandong First Medical University and Shandong Academy of Medical Sciences, Jinan, People's Republic of China

Tel +86 I5I-54I8-0386

Fax +8631 67626624

Emailboc_III@I63.com
Purpose: The Y-box binding protein $1(Y B X 1)$ gene encodes the multifunctional protein YB1 that is associated with the dysregulation of numerous cancer-related genes. However, the prognostic value of $Y B X 1$ and its correlation with immune cell infiltration in breast cancer (BRCA) remain unclear.

Methods: $Y B X 1$ expression data in various malignancies were obtained from Oncomine, Tumor Immune Estimation Resource (TIMER), Cancer Cell Line Encyclopedia, UALCAN and cBio Cancer Genomics Portal databases. Survival data were analyzed with KaplanMeier plotter. Immune cell infiltration and its association with $Y B X 1$ expression level were assessed with TIMER and LinkedOmics. YB1 expression was evaluated by immunohistochemistry and Western blotting, and changes in cancer cell viability and $\mathrm{T}$ cell activity following $Y B X 1$ knockdown were assessed with an immunocyte-tumor cell co-culture assay. Results: $Y B X 1$ was downregulated in the BRCA cohort, which was closely associated with worse prognosis in the luminal A subtype (overall survival [OS]: hazard ratio [HR] 1.93, 95\% confidence interval [CI] 1.22-3.05, $P=0.0042$; recurrence-free survival [RFS]: HR $1.85,95 \%$ CI $1.51-2.28, P=3.1 \mathrm{e}-9$ ) and luminal B subtype (OS: HR 1.08, 95\% CI 0.68 $1.70, P=0.75$; RFS: HR $1.29,95 \%$ CI $1.02-1.62, P=0.03) . Y B X 1$ expression was positively correlated with the M2 macrophage infiltration and expression of T cell exhaustion markers such as indoleamine 2,3-dioxygenase 1 (IDO1) $(\mathrm{rs}=0.388, P=4.93 \mathrm{e}-37)$ and cytotoxic T-lymphocyte-associated protein 4 (CTLA4) $(\mathrm{rs}=0.321, P=2.54 \mathrm{e}-25)$ in luminal BRCA. Kaplan-Meier analysis revealed a correlation between $Y B X 1$ expression, M2 infiltration and survival outcome. Co-culture with macrophages or T cells enhanced the decrease in luminal BRCA cell viability induced by $Y B X 1$ knockdown.

Conclusion: High $Y B X 1 \mathrm{mRNA}$ levels predict a poor prognosis in luminal BRCA, which is correlated with M2 macrophage infiltration and T cell exhaustion in the tumor microenvironment. Combining classic therapeutics with immune checkpoint inhibitors and M1 polarization agents may be an effective treatment strategy for luminal BRCA with $Y B X 1$ overexpression.

Keywords: Y-box binding protein 1, breast cancer, prognosis, immune cell infiltration, macrophages, immunosuppression, 3D co-culture

\section{Introduction}

Breast cancer (BRCA) is the leading cause of new cancer diagnoses and the second leading cause of cancer-related deaths among women worldwide. ${ }^{1}$ In China, BRCA accounts for $12.2 \%$ of all new diagnoses and $9.6 \%$ of all deaths of tumor patients, 
respectively. ${ }^{2}$ With early screening and improvements in treatment, the 5-year survival rate of BRCA is now over $85 \%{ }^{3,4}$ However, there are still some subtypes, such as triple negative BRCA (TNBC) and metastatic BRCA, carrying poor prognosis. ${ }^{5,6}$ To further improve treatment efficacy especially for the refractory subtypes above, one major step is to elucidating the genomic and molecular signatures of BRCA, which can help to identify high-risk patients thereby administering optimal therapies. ${ }^{4,7}$ Currently, public databases offer large amounts of data that can facilitate this endeavor.

Located at $1 \mathrm{p} 34.2$, the gene Y-box binding protein 1 $(Y B X 1)$ encodes a $36 \mathrm{kDa}$ DNA/RNA binding protein, namely YB1, containing a highly conserved cold shock domain. ${ }^{8}$ More than $90 \%$ of YB1 distributes in the cytoplasm, ${ }^{9}$ where it regulates translation by directly binding to messenger RNA (mRNA); ${ }^{10}$ while in response to noxious stimuli including radiation and DNA damaging agents (eg, cisplatin and mitomycin $\mathrm{C}$ ), ${ }^{11}$ cytoplasmic YB1 translocates into the nucleus, binding the target promoters with the so-called Y-box sequence (ie, an inverted CCAAT box) and regulating transcription. ${ }^{12}$ Clinically, $Y B X 1$ overexpression was shown to be an independent prognostic biomarker in BRCA, ${ }^{13,14}$ and it has been experimentally explained by the enhancement of epithelial-to-mesenchymal transition (EMT) ${ }^{15,16}$ and the induction of chemoresistance ${ }^{17,18}$ in cancer cells. However, little is known about the impact of $Y B X 1$ on immune infiltrates in tumor microenvironment. Recent studies have demonstrated an association between $Y B X 1$ and immunologic processes such as inflammation and activation of innate immunity in non-neoplastic disorders. ${ }^{19,20}$ Therefore, we speculate that changes in $Y B X 1$ expression modulate the tumor microenvironment in BRCA.

To test this hypothesis, in the present study we evaluated the expression pattern of $Y B X 1$ and its relationship to prognosis and immune cell infiltration in BRCA through analysis of public datasets and in vitro experiments. Firstly, we observed the significantly distinct expression of $Y B X 1$ in different BRCA subtypes by mRNA level evaluation in the UALCAN database, the immunohistochemical staining in breast samples and the protein assays in multiple breast cell lines. Then, the Kaplan-Meier plotters showed the significantly poorer survival outcomes in $Y B X 1$ high-expressing groups compared with the low ones in BRCA especially with luminal subtypes. Furthermore, we evaluated the infiltration levels of various immune cells with increasing expression of $Y B X 1$ via the databases such as LinkedOmics and Tumor Immune Estimation Resource (TIMER), finding the significant positive correlation of $Y B X 1$ with macrophage (M2) infiltration and $\mathrm{T}$ cell exhaustion markers such as IDOI (indoleamine 2,3-dioxygenase 1), CTLA4 (cytotoxic T-lymphocyte associated protein 4), in luminal BRCA, which was verified by the tumor inhibition after $Y B X I$ knockdown in the co-culture system of cancer cells and immunocytes. The findings in this report pioneered the explanation for the poorer prognosis of BRCA with high $Y B X 1$ expression from the point of view of tumor microenvironment, and shed light on the better treatment outcomes of IDO1/CTLA4 inhibitors in $Y B X 1$ high-expressing luminal BRCA patients.

\section{Materials and Methods Oncomine Database Analysis}

The mRNA expression of $Y B X 1$ in various cancer types was identified via the Oncomine database (https://www. oncomine.org). ${ }^{21}$ Oncomine is the largest oncogene database covering 715 datasets and 86,733 samples. $^{21}$ We focused on the comparison of $Y B X 1$ expression between BRCA and normal tissues and set the thresholds as follows: $P$-value: 0.01 ; fold change: 1.5 ; gene rank: $10 \%$.

\section{Cancer Cell Line Encyclopedia (CCLE) Analysis}

CCLE is a large genomic and proteomic database of human cancer cell lines generated by the Broad Institute and Novartis. ${ }^{22}$ To verify the findings from the Oncomine database, the protein expression of YB1 in cell lines of various cancer types was identified by CCLE.

\section{UALCAN Analysis}

The relative expression of $Y B X 1$ across diverse BRCA subgroups was evaluated via UALCAN database (http:// ualcan.path.uab.edu), a comprehensive interactive webportal for analyzing tumor subgroup gene expression. ${ }^{23}$ The gene expression and clinical data of UALCAN are derived from The Cancer Genome Atlas (TCGA).

\section{The cBio Cancer Genomics Portal (cBioPortal) Database Analysis}

The cBioPortal database (https://www.cbioportal.org) is an open-access web-based platform for interactive exploration of multidimensional cancer genomics data. ${ }^{24,25} \mathrm{We}$ selected the largest dataset of BRCA in cBioPortal, Breast Cancer (METABRIC, Nature 2012 and Nat 
Commun 2016) with 2509 primary BRCA samples, for the following analysis. The module of "Oncoprint" shows the overview of $Y B X 1$ alterations in BRCA cases, which we used to investigate the changes of clinical parameters including age, histologic grade, tumor stage etc with the increase of $Y B X 1$ expression in BRCA. The module of "Co-expression" evaluates the correlation between different genes in BRCA and we used it to verify the significant correlation of $Y B X 1$ with immunosuppression-associated genes including IDO1, PDCD1, CTLA4 and LAG3.

\section{Kaplan-Meier Plotter Database Analysis}

Aggregating data from Gene Expression Omnibus (GEO), European Genome-phenome Archive (EGA), and TCGA, the Kaplan-Meier plotter database can assess the effect of more than 54,000 genes (mRNA, miRNA, protein) on survival especially in BRCA $(n=6234) .{ }^{26}$ The correlation of $Y B X 1$ expression with survival in BRCA as well as in BRCA subgroups divided by various clinicopathological characteristics was analyzed by Kaplan-Meier plotter (http://kmplot.com/analysis). Kaplan-Meier plots of overall survival (OS) and relapse-free survival (RFS) were drawn, respectively, and the hazard ratio (HR) with $95 \%$ confidence intervals $(95 \% \mathrm{CI})$ and log-rank $P$-value were also calculated.

\section{TIMER Database Analysis}

TIMER is a comprehensive web-portal for analyzing immune infiltration systemically across various cancer types (http://timer.cistrome.org). ${ }^{27-29}$ We applied multiple modules in the TIMER database for the following evaluation: (a) the "Exploration-Gene_DE" module for evaluating the differential expression of $Y B X 1$ between tumor and adjacent normal tissues across all TCGA tumors; (b) the "Gene_Outcome" module for verifying the independent prognostic role of $Y B X 1$ expression in BRCA after adjusting potential confounding factors; (c) the "Immune-Gene" module for evaluating the correlation of $Y B X 1$ expression with the abundance of immune infiltrates, including $\mathrm{B}$ cells, CD4 $+\mathrm{T}$ cells, CD8 $+\mathrm{T}$ cells, macrophages, and myeloid-derived suppressor cells; (d) the "ExplorationGene_Corr" module for evaluating the correlation between $Y B X 1$ and gene markers of tumor-infiltrating immune cells; (e) the "Immune-Outcome" module for evaluating the clinical relevance of macrophage subsets, M1 and M2, along with different expression of $Y B X 1$ in $\mathrm{BRCA} ;{ }^{30}$ (f) the "Estimation" module for evaluating the relative infiltration levels of macrophage subsets from
GSE1456 GPL96, a representative dataset for BRCA patients. ${ }^{31,32}$ The data of GSE1456_GPL96 were obtained from https://www.ncbi.nlm.nih.gov/geo.

\section{LinkedOmics Analysis}

LinkedOmics database (http://www.linkedomics.org/login. php) is an open-access tool containing multi-omics data from all 32 TCGA cancer types. ${ }^{33}$ The module of "LinkFinder" in this database can be applied to searching for attributes associated with a query attribute including mRNA expression signatures of genomic alterations etc. We used it to find the immunosuppression-associated genes that have a significant positive correlation with YBX1 via the dataset of TCGA_BRCA (HiSeq RNA).

\section{Cell Lines and Antibodies}

Human breast epithelial MCF-10A cells and human BRCA cells (MCF-7, BT-474, SK-BR-3, MDA-MB-231) were purchased from the Cell Bank of the Chinese Academy of Science (Shanghai, China), and human BRCA cells namely HCC1428, HCC1569 and HCC1143 were purchased from the American Type Culture Collection (ATCC, Manassas, VA, USA). All the cells were cultured at $37^{\circ} \mathrm{C}$ in Dulbecco's modified Eagle's medium (DMEM) (Gibco, Thermo Fisher Scientific, Inc., Waltham, MA, USA) supplemented with $10 \%$ fetal bovine serum (FBS; Gibco, Thermo Fisher Scientific, Inc.), 100U/ $\mathrm{mL}$ penicillin, and $100 \mu \mathrm{g} / \mathrm{mL}$ streptomycin in a humidified atmosphere of $5 \% \mathrm{CO}_{2}$.

The antibodies used in this study include: YB1 (\#ab76149; Abcam, Cambridge, MA, USA), IDO1 (\#13268-1-AP; Proteintech, Chicago, USA), CTLA4 (\#ab237712; Abcam, Cambridge, MA, USA), and $\beta$-actin (\#ab8226; Abcam, Cambridge, MA, USA).

\section{Immunohistochemistry}

YB1, IDO1 and CTLA4 staining were conducted with formalin-fixed, paraffin-embedded breast samples. Pathological sectioning was performed by Jujie Sun, Department of Pathology, Shandong Cancer Hospital and Institute, Shandong First Medical University and Shandong Academy of Medical Sciences. Fivemicrometer-thick sections were deparaffinized within xylene, rehydrated in graded ethanol, and washed thrice with threefold-distilled water. For antigen retrieval, sections were incubated within Tris-EDTA buffer ( $\mathrm{pH} 9.0$ ), heated at $120^{\circ} \mathrm{C}$ for $10 \mathrm{~min}$, and cooled down to room temperature (RT). After incubation with primary 
antibodies (1:100 dilution for anti-IDO1 and anti-YB1 antibodies, 1:150 dilution for anti-CTLA4 antibody) overnight at $4^{\circ} \mathrm{C}$, the samples were then rinsed with phosphate buffered saline (PBS) thrice, incubated with goat antirabbit IgG secondary antibodies (A0208, 1:50 dilution; Beyotime Institute of Biotechnology, China) for $50 \mathrm{~min}-$ utes at RT. After washed with PBS again and stained with diaminobenzidine (DAB), the sections were then counterstained with Mayer's hematoxylin, dehydrated, and mounted. The pathological images were obtained with a high-resolution scanner. The number of immunohistochemical positive cells and total cells were counted in three non-overlapping fields per section by two pathologists independently and percentage of positive cells was calculated. All the manipulations were approved by the Ethic Committee of Shandong Cancer Hospital and Institute.

\section{Protein Extraction and Western Blotting}

Protein extraction and Western blotting assay were performed as previously described. ${ }^{34}$ Breast cells after different treatments were washed with ice-cold PBS thrice. RIPA lysis buffer (P0013B, Beyotime Institute of Biotechnology, China) with $1 \mathrm{mM}$ phenylmethanesulfonyl fluoride solution (PMSF) was used for cell lysis and total protein extraction. Protein extracts were boiled at $100^{\circ} \mathrm{C}$ for 15 minutes, followed by separated with $10 \%$ sodium dodecyl sulfate polyacrylamide gel electrophoresis (SDSPAGE) and then transferred into the nitrocellulose (NC) membrane (Millipore, Billerica, MA, USA). Membrane was blocked by $5 \%$ skimmed milk at RT for one hour. After the incubation with primary antibodies overnight at $4^{\circ} \mathrm{C}$, the blots were then incubated with corresponding goat anti-rabbit (A0208, 1:2000; Beyotime Institute of Biotechnology, China) or goat anti-mouse (A0216, 1:2000; Beyotime Institute of Biotechnology, China) IgG horseradish peroxidase-conjugated secondary antibodies for 45 minutes at RT. Then, the membrane was washed using Tris-buffered saline with Tween-20 buffer (TBST) thrice for 7 minutes at each time. The signals were detected using electrochemiluminescence substrates (Millipore, Billerica, MA, USA).

\section{Isolation of Macrophages}

Murine peritoneal macrophage cells were isolated from eight-week old female BALB/C mice (the Institute of Zoology, Chinese Academy of Sciences, Beijing, China). Briefly, 3\% Brewer thioglycollate medium $(1 \mathrm{~mL}$ per mouse daily, for 3 days) was injected into the peritoneal cavity 4 days before euthanization of the mice. After euthanized by rapid cervical dislocation, the mice were subjected to laparotomy by making an incision along the midline. Intraperitoneal injection with ice-cold PBS was performed by $10-\mathrm{mL}$ syringe, and then the fluid was collected into a $50-\mathrm{mL}$ centrifuge tube for further centrifugation at $400 \times \mathrm{g}$, for 10 minutes, at $4^{\circ} \mathrm{C}$. The cell pellet was resuspended gently by ice-cold DMEM/F12 with $10 \%$ FBS and seeded into a 6 -well cell culture plate $\left(2 \times 10^{6}\right.$ cells per well). After 24 hours, the non-adherent cells were removed by gently washing with PBS. The adherent macrophages were subjected to $3 \mathrm{D}$ co-culture assay.

\section{D Co-Culture of BRCA Cells and Macrophages}

To establish a 3D co-culture system, 50\% Matrigel (Matrigel: DMEM=1:1) was added into 96-well plates ( $45 \mu \mathrm{L}$ per well) and incubated for 100 minutes at $37^{\circ} \mathrm{C}$ in a humidified atmosphere of 5\% CO2. MCF-7 (7000 cells per well), BT-474 (11,000 cells per well) or MDA-MB-231 (7000 cells per well) after transfected with $Y B X 1$ small interfering RNA (siRNA) or scrambled siRNA for 24 hours were mixed with peritoneal macrophage cells (7000 cells per well) in the pre-coated 96-well plate, and co-cultured using DMEM with $10 \%$ FBS and $2 \%$ matrigel for another 48 hours.

\section{CCK-8 Assay}

A cell counting kit-8 (CCK-8; CK04-500T, Dojindo Laboratories Co. Ltd, Kumamoto, Japan) was applied to assess cell viability. 5\% CCK-8 solution (CCK-8: DMEM=5:95) was added into 96-well plates $(100 \mathrm{~mL}$ per well), and the absorbance at $450 \mathrm{~nm}$ was assessed by a multilabel counter after 15-60 minutes of incubation in dark at $37^{\circ} \mathrm{C}$.

\section{Isolation of CD8+ T Cells}

Firstly, mononuclear cells were isolated from peripheral blood samples of healthy adult donors by Ficoll-Paque PLUS (Cytiva, Marlborough, MA, USA) density gradient centrifugation. Cell extracts were incubated with anti-CD8 antibody-coated magnetic microbeads (\#130-045-201, Miltenyi Biotec, Bergisch Gladbach, Germany) for 15 min at $4{ }^{\circ} \mathrm{C}$, and then loaded onto a magnetic activated cell sorter (MACS) column that was placed in the magnetic field of the OctoMACS separator (\#130-024-109, Miltenyi Biotec, Bergisch Gladbach, Germany). After 
washes with PBS, the column was removed from the magnetic field and CD8+ T cells were eluted.

\section{Enzyme-Linked Immunosorbent Assay (ELISA) for Co-Culture Cytokines}

To evaluate the interaction between BRCA cells and CD8+ $\mathrm{T}$ cells, the co-culture systems were established. Firstly, the $\mathrm{CD} 8+\mathrm{T}$ cells were stimulated with $3 \mathrm{ng} / \mathrm{mL}$ of phorbol $12-$ myristate 13 -acetate (PMA) and $1 \mu \mathrm{g} / \mathrm{mL}$ of ionomycin, and the cancer cells were transfected by siRNA. Then, the activated CD8+ T cells and the transfected cancer cells were cocultured in $35 \mathrm{~mm}$ cell culture dishes at a ratio of 5: 1 for 48 hours. The culture medium was collected after centrifugation at $2500 \mathrm{rpm}$ for 20 minutes, and the secreted interleukin-2 (IL-2) and interferon-gamma (IFN- $\gamma$ ) in the medium were measured by ELISA kits (Mlbio, Shanghai, China) according to the manufacturer's instructions.

\section{Assay for CD8+ T Cell Cytotoxicity to BRCA Cells}

MCF-7, BT-474, or MDA-MB-231 cells after siRNA transfection were co-cultured with activated CD8+ T cells for 48 hours (cancer cells: $\mathrm{T}$ cells $=5: 1$ ). The cytotoxicity of CD8+ $\mathrm{T}$ cells was determined by measuring lactate dehydrogenase (LDH) release from damaged cells using LDH Cytotoxicity Assay Kit (C0016; Beyotime Institute of Biotechnology, Shanghai, China). According to the manufacturer's protocol. Briefly, the co-culture plate was centrifuged at $400 \times \mathrm{g}$ for 5 minutes, and $120 \mu \mathrm{L}$ of supernatant from each well was aspirated and transferred to a new well and mixed with $60 \mu \mathrm{L}$ of $\mathrm{LDH}$ detection reagent. After incubation at RT for 30 minutes, the absorbance at $490 \mathrm{~nm}$ and $600 \mathrm{~nm}$ was measured with a multi-label counter and the amount of released LDH was calculated.

\section{siRNA Interference and Transfections}

$Y B X 1$-siRNA was purchased from GenePharma (Shanghai, China). The targeting sequences were as follows (gene: sense, antisense). YBX1 (\#1): 5'-CGUAACCAUUA UAGACGCUTT-3'， 5'-AGCGUCUAUAAUGGUUACG TT-3'; YBX1 (\#2): 5'-GGAUAUGGUUUCAUCAACATT -3', 5'-UGUUGAUGAAACCAUAUCCTT-3'; negative control: 5'-UUCUCCGAACGUGUCACGUTT-3', 5'ACGUGACACGUUCGGAGAATT-3'. BRCA cells were seeded into six-well plates generally with a confluence of $30 \%$. Transfections without serum were conducted with 80nM YBXI siRNA or scrambled siRNAs using
Lipofectamine 2000 reagent (Invitrogen, Carlsbad, CA) for 6 hours. After additional 24-hour culture with serum, the cells were subjected to the above-mentioned assays.

\section{Statistical Analysis}

The results generated in Oncomine are displayed with $P$-values and median rank. The Kaplan-Meier survival curves are displayed with $\mathrm{HR}$ and $P$-values from a $\log$ rank test. The correlation between different genes was evaluated by the Spearman correlation test. In cellular experiments, data in bar graphs are expressed as the mean \pm standard deviation (SD) from at least three independent experiments. Statistical analysis was conducted with the Student's unpaired $t$-test or one-way analysis of variance (ANOVA). A $P$-values $<0.05$ were considered statistically significant.

\section{Results}

\section{Decreased Expression of $Y B X I$ in BRCA} We examined $Y B X 1 \mathrm{mRNA}$ levels in various cancers in the Oncomine database. The mRNA expression of $Y B X 1$ was lower in bladder, esophageal, and lung cancers, leukemia, myeloma, sarcoma, and especially in BRCA compared with the corresponding normal tissues; while higher expression of $Y B X 1$ was observed in bladder, brain, cervical, esophageal, head and neck, kidney, and ovarian cancers, myeloma, sarcoma and lymphoma (Figure 1A and B). RNA sequencing data from TIMER verified the findings by showing the significantly lower levels of $Y B X 1$ in BRCA, and the significantly higher ones in ESCA (esophageal carcinoma), HNSC (head and neck squamous cell carcinoma), KICH (kidney chromophobe cell carcinoma), LIHC (liver hepatocellular carcinoma), and STAD (stomach adenocarcinoma) (Figure 1C). Additionally, the protein array from CCLE database revealed a mean YB1 protein level below the baseline in BRCA cells, which also indicated the decreased expression of $Y B X 1$ in BRCA (Figure 1D).

\section{YBXI Level is Higher in BRCA Subtypes with Worse Prognosis}

To further understand the expression pattern of $Y B X 1$, BRCA samples were divided into subgroups according to clinicopathological features (Figure 2A-H). The UALCAN analysis showed that $Y B X 1$ expression decreased along with patient age (Figure 2B). Moreover, despite the decreased levels in the overall BRCA patient population, $Y B X 1$ was overexpressed particularly in the 
A

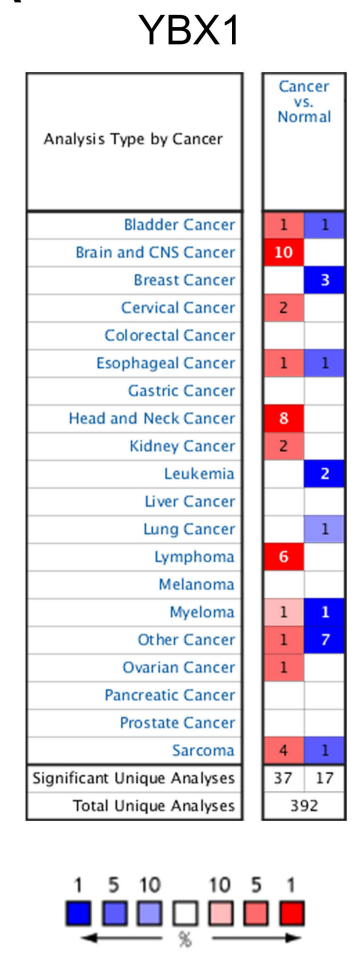

B

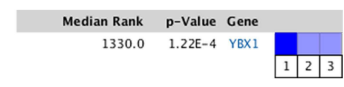

1. Invasive Breast Carcinoma Stroma vs. Normal Finak Breast, Nat Med, 2008

2. Intraductal Cribriform Breast Adenocarcinoma vs. Normal TCGA Breast, No Associated Paper, 2011

3. Mixed Lobular and Ductal Breast Carcinoma vs. Norma

TCGA Breast, No Associated Paper, 2011
C

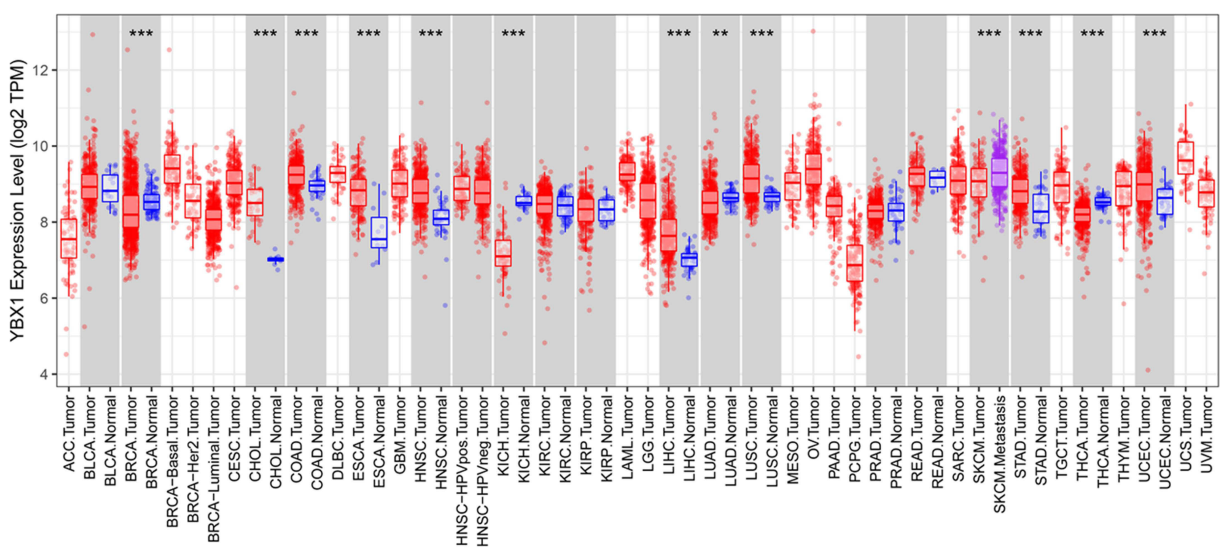

D
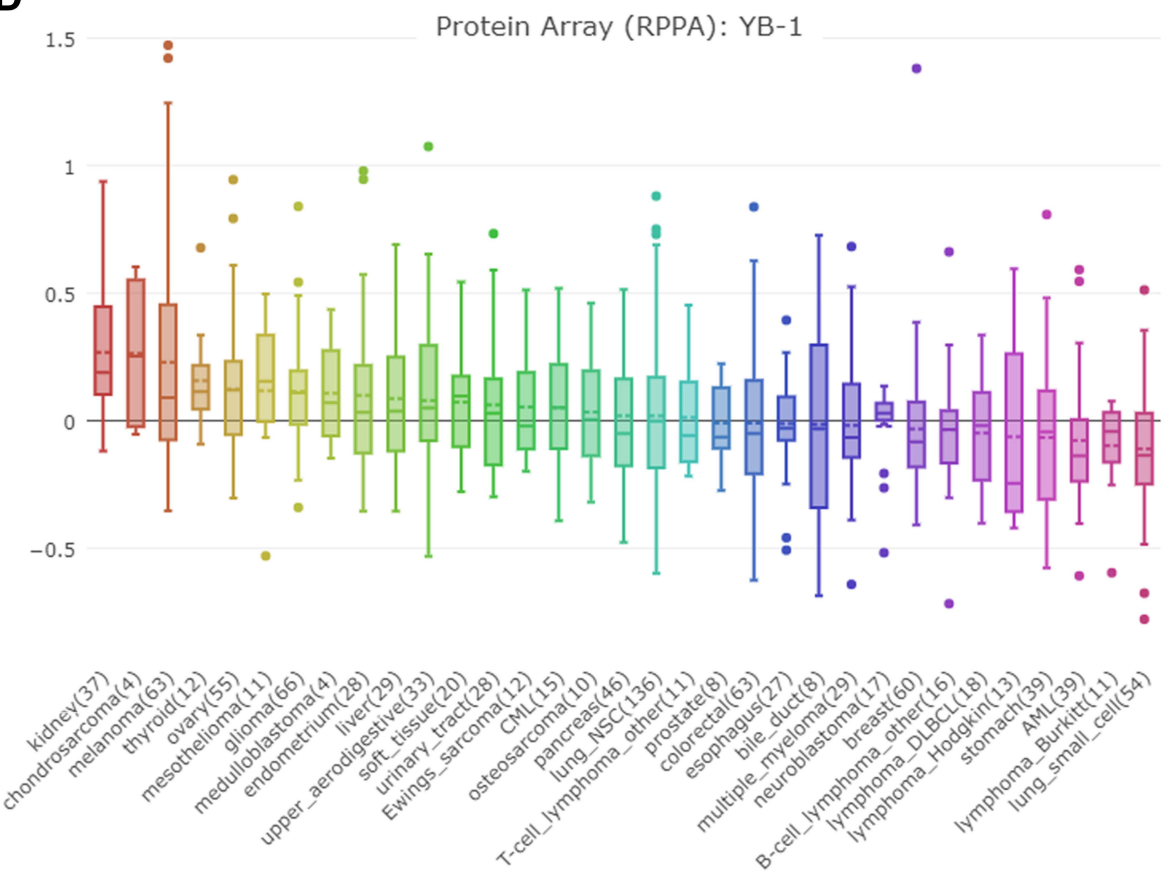

Figure I The YBXI expression profiles in different type of human cancers.

Notes: (A) Increased or decreased mRNA levels of $Y B X I$ in datasets of various cancers compared with normal tissues via Oncomine database. (B) Representative datasets of BRCA with decreased YBXI expression compared with normal tissues in Oncomine. (C) The expression levels of YBXI in different tumor types analyzed by TIMER database. (D) The protein levels of $Y B X I$ in different type of cancer cell lines. $* * P<0.01, * * * P<0.001$ compared with corresponding normal tissues.

Abbreviations: BRCA, breast cancer; RPPA, reverse phase protein arrays; TIMER, the Tumor Immune Estimation Resource database; YBXI, Y-box binding protein I.

genomic subtype of TP53-mutant BRCA (Figure 2E), and in the molecular subtype of TNBC (Figure $2 \mathrm{~F}$ and $\mathrm{G}$ ) which were known to carrying worse prognosis. ${ }^{35}$ However, there was no exact relationship between $Y B X 1$ expression and tumor stage or nodal metastasis status (Figure 2D and $\mathrm{H}$ ). To verify these findings, the clustering of several clinicopathological parameters was performed according to the $Y B X 1$ mRNA level using cBioPortal database, and the BRCA with high histologic grade, as well as the TNBC, were clustered to the side of high
YBX1 expression (Figure 3A). Besides, immunohistochemical and Western blot analyses of YB1 in different molecular subtypes of BRCA also revealed that YB1 expression was highest in TNBC (Figure 3B and C).

\section{High Expression of YBXI Predicts Shorter Survival in BRCA Patients}

Survival data of BRCA from GEO, EGA, and TCGA were meta-analyzed using the Kaplan-Meier plotter. For the entire BRCA population, the OS and RFS in YBXI high-expressing 
A

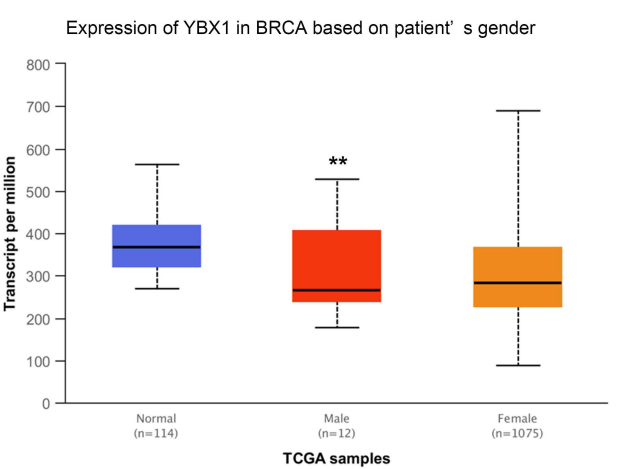

B

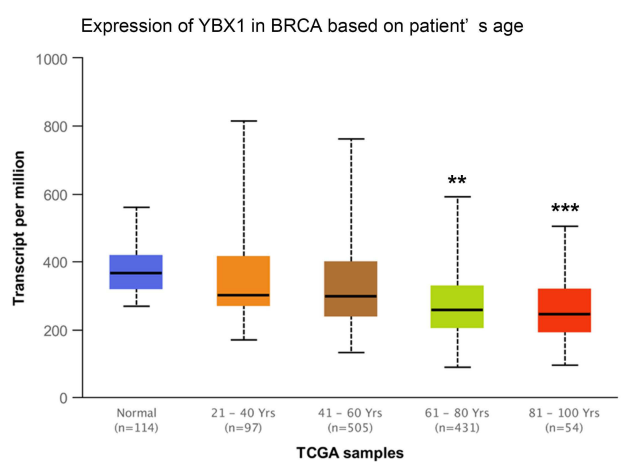

C

Expression of YBX1 in BRCA based on histologic subtypes

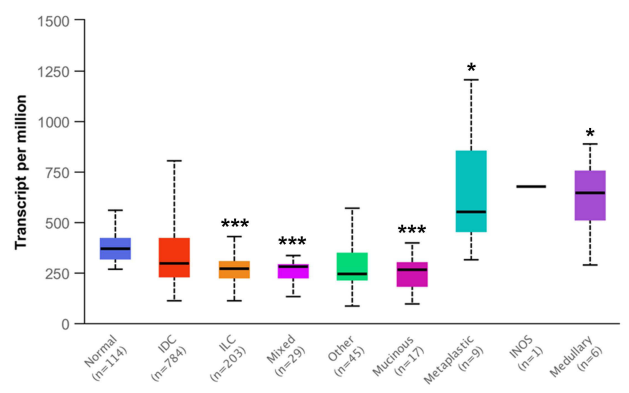

TCGA samples

\section{D}

Expression of YBX1 in BRCA based on individual cancer stages

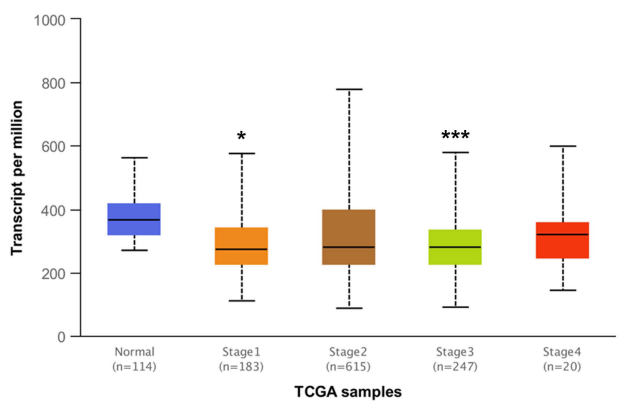

E

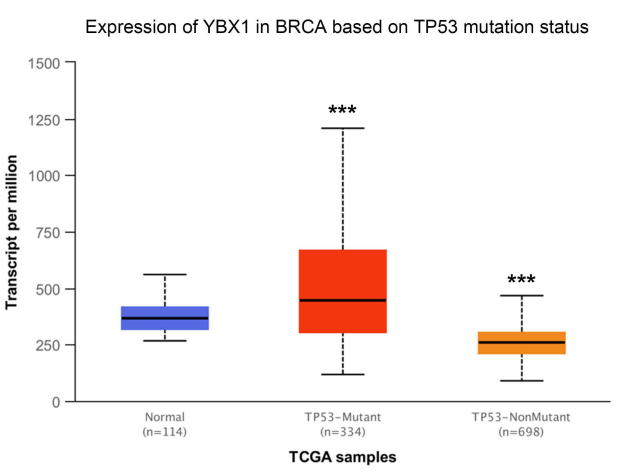

$\mathbf{F}$

Expression of YBX1 in BRCA based on breast cancer sybclasses

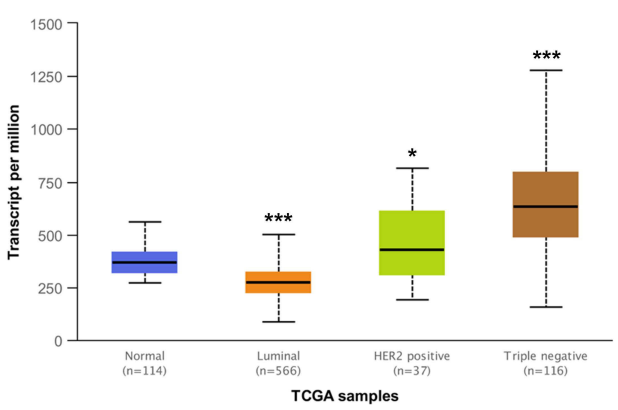

G

Expression of YBX1 in BRCA based on Major subclasses (with TNBC types)

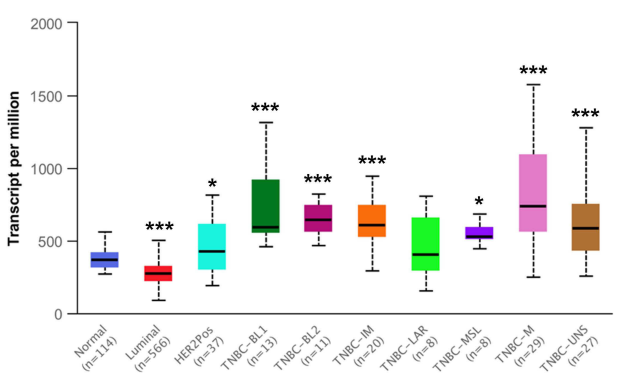

TCGA samples

H

Expression of YBX1 in BRCA based on nodal metastasis status

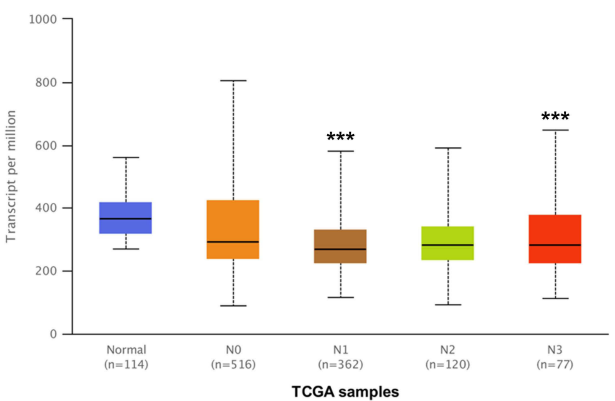

Figure 2 Subgroup analysis of $Y B X I$ mRNA level in BRCA by UALCAN database.

Notes: Boxplot for relative mRNA levels of YBXI in normal tissues and BRCA samples with different (A) genders; (B) age stages; (C) histologic subtypes; (D) cancer stages; (E) TP53 mutation status; (F) molecular subtypes; (G) triple-negative BRCA subtypes; $(\mathbf{H})$ nodal metastasis status. $* P<0.05$, $* * P<0.0$ I, $* * * P<0.00$ I compared with normal tissues.

Abbreviations: BRCA, breast cancer; IDC, invasive ductal carcinoma; ILC, invasive lobular carcinoma; INOS, infiltrating- not otherwise specified; TCGA, The Cancer Genome Atlas; TP53, tumor protein 53; YBXI, Y-box binding protein I. 
group were significantly lower than those in $Y B X 1$ lowexpressing one (OS: HR 1.67, 95\% CI 1.27-2.18, $P=0.00017$; RFS: HR 1.83 , 95\% CI 1.61-2.09, $P=1 \mathrm{e}-16$; Figure $4 \mathrm{~A}$ and $\mathrm{B})$. The difference in survival outcomes remained significant after adjustment for potential confounds including patient age, sex, ethnicity and tumor stage using the TIMER tool (Supplementary Figure 1). To determine whether the worse outcomes of the $Y B X 1$ high-expressing group was due to the poorer prognosis of the TNBC subtype, we evaluated the OS and RFS in the four molecular subtypes of BRCA (luminal A, luminal B, TNBC, and human epidermal growth factor receptor 2 [HER2]-enriched). Interestingly, the significantly lower survival rates of $Y B X 1$ high-expressing groups were demonstrated not in the HER2enriched or TNBC subtypes, but in the two luminal subtypes (luminal A: OS: HR 1.93, 95\% CI 1.22-3.05, $P=0.0042$; RFS: HR 1.85, 95\% CI 1.51-2.28, $P=3.1 \mathrm{e}-9$; luminal B: OS: HR 1.08, 95\% CI 0.68-1.70, $P=0.75$; RFS: HR 1.29 , 95\% CI 1.02-1.62, $P=0.03$; HER2-enriched: OS: HR 0.90, 95\% CI $0.43-1.86, P=0.77$; RFS: HR $0.90,95 \%$ CI $0.57-$ 1.43, $P=0.65$; TNBC: OS: HR $1.44,95 \%$ CI $0.80-2.58$, $P=0.22 ;$ RFS: HR $1.10,95 \%$ CI $0.81-1.50, P=0.53$; Table 1, Figure 4A and B). Taken together, these results suggest the independent prognostic value of $Y B X 1$ in BRCA rather than the impact of confounding factors or the overexpression of $Y B X 1$ in TNBC.

To better understand potential mechanisms of the poorer prognosis in $Y B X 1$ high-expressing BRCA, we analyzed the correlation between $Y B X 1$ level and patients' clinicopathologic characteristics based on data in the Kaplan-Meier plotter database. High expression of $Y B X 1$ was associated with shorter RFS in estrogen receptor (ER)-positive patients (HR $1.24,95 \% \mathrm{CI} 1.06-1.47, P=0.0088)$ as well as HER2-negative patients (HR 1.84, 95\% CI 1.41-2.41, $P=6.4 \mathrm{e}-6$; Table 1), justifying the worse prognosis of luminal BRCA with high $Y B X 1$ expression. In particular, a stronger correlation was observed between $Y B X 1$ overexpression and negative lymph node status (OS: $\mathrm{HR}=1.79,95 \%$ CI: $1.22-2.62, \mathrm{P}=0.0025$ ) as compared to positive lymph node status (OS: HR 1.05, 95\% CI $0.71-1.55, P=0.80$; Table 1). This finding suggests that $Y B X 1$ expression level has a greater impact on the prognosis of BRCA patients without lymph node metastasis.

\section{YBXI is Correlated with Macrophage Polarization from $\mathrm{MI}$ to $\mathrm{M} 2$ in BRCA}

Immune infiltrates play an important role in predicting clinical outcomes in various cancers including
BRCA. ${ }^{36,37}$ Here, we evaluated the association between $Y B X 1$ and immune cell infiltration in BRCA via the TIMER database. For the entire BRCA population as well as the four molecular subtypes except for TNBC, $Y B X 1$ was significantly negative correlated with tumor purity (BRCA: rs $=-0.155, P=8.27 \mathrm{e}-7$; BRCA-luminal A: $\mathrm{rs}=-0.225, P=3.85 \mathrm{e}-9$; BRCA-luminall $\mathrm{B}: \mathrm{rs}=-0.195$, $P=6.48 \mathrm{e}-3$; BRCA-HER2: $\mathrm{rs}=-0.241, P=3.98 \mathrm{e}-2$; BRCATNBC: $\mathrm{rs}=0.167, P=2.69 \mathrm{e}-2$; Figure $5 \mathrm{~A}-\mathrm{E}$ ), suggesting the increasing immune cell infiltration along with increasing expression of $Y B X 1$. When we examined individual infiltrating cell types, we found a significant positive correlation between $Y B X 1$ and macrophages in the entire BRCA population and in patients with the luminal subtypes but not in HER2-enriched and TNBC patients (BRCA: $\quad$ rs $=0.222, \quad P=1.50 \mathrm{e}-12 ; \quad$ BRCA-luminal A: rs $=0.224, \quad P=2.81 \mathrm{e}-7$; $\quad$ BRCA-luminal $\quad B: \quad r s=0.257$, $P=3.29 \mathrm{e}-4$; BRCA-HER 2 : $\mathrm{rs}=-0.040, P=7.36 \mathrm{e}-1$; BRCATNBC: rs $=0.024, P=7.56 \mathrm{e}-1)$. As macrophages in the tumor microenvironment can differentiate into M1 and M2 which possess opposite effects on tumor progression, we examined the relationship between the expression of M1 and M2 macrophage markers and YBX1 in the TIMER database. The results showed that the gene markers of M2 macrophages, namely CD163 (rs $=0.289$, $P=1.54 \mathrm{e}-20), \quad$ VSIG4 $\quad(\mathrm{rs}=0.184, \quad P=4.69 \mathrm{e}-9), \quad$ and MS4A4A (rs=0.220, $P=2.54 \mathrm{e}-12$ ) had stronger positive correlations with $Y B X 1$ expression than the M1 macrophage markers (iNOS: rs $=0.198, P=2.75 \mathrm{e}-10$; IRF5: rs $=0.061, P=5.62 \mathrm{e}-2$; COX2: $\mathrm{rs}=0.243, P=8.91 \mathrm{e}-15)$ in BRCA (Table 2). Moreover, the tendency was more obvious in BRCA with luminal subtypes (luminal A: CD163: $\quad$ rs $=0.271, \quad P=1.45 \mathrm{e}-4 ; \quad$ VSIG4: $\quad \mathrm{rs}=0.159$, $P=2.78 \mathrm{e}-2 ; \quad$ MS4A4A： rs $=0.189, \quad P=8.82 \mathrm{e}-3 ; \quad$ iNOS: rs $=0.060, \quad P=1.74 \mathrm{e}-1 ; \quad$ IRF5: $\quad$ rs $=0.046, \quad P=2.93 \mathrm{e}-1$; COX2: rs $=-0.060, \quad P=4.10 \mathrm{e}-1$; luminal $\mathrm{B}: \mathrm{CD} 163$ : rs $=0.271, \quad P=1.45 \mathrm{e}-4 ; \quad$ VSIG4: $\quad \mathrm{rs}=0.159, \quad P=2.78 \mathrm{e}-2$; MS4A4A: $\quad \mathrm{rs}=0.189, P=8.82 \mathrm{e}-3$; iNOS: $\mathrm{rs}=0.084$, $P=2.47 \mathrm{e}-1$; IRF5: $\mathrm{rs}=-0.039, P=5.90 \mathrm{e}-1 ; \mathrm{COX} 2$ : $\mathrm{rs}=$ $-0.060, P=4.10 \mathrm{e}-1$; Table 2).

To validate the above findings, we analyzed data in the TIMER database and performed a macrophage-tumor cell co-culture assay. In the TIMER analysis, the immune infiltration estimation with GSE1456 dataset reviewed remarkable increases of M0 and M2, rather than M1, macrophage infiltration in $Y B X 1$ high-expressing group (Figure 6A). Moreover, when analyzing survival outcomes in relation to macrophage infiltration and $Y B X 1$ expression in BRCA 
A

Mutation spectrum

Age at diagnosis

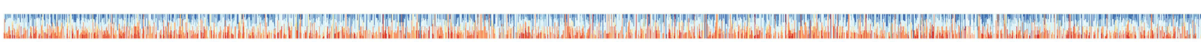

Pam50+Claudin-low subtype

Neoplasm histologic grade

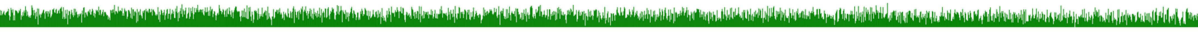

Tumor stage

|

Lymph nodes examined positive

Cancer type detailed

Tumor other histologic subtype

YBX1 mRNA expression

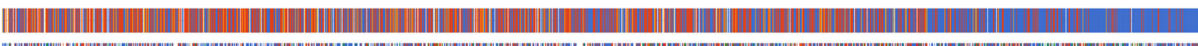

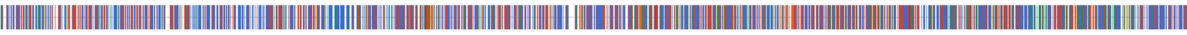

\begin{tabular}{|c|c|}
\hline Mutation spectrum & $\begin{array}{llllllll}\| & \| A & \| C>G & C>T & \| T>A & \| T>C & \| T>G & - \text { Nodata }\end{array}$ \\
\hline Age at diagnosis & $21.92 / 96.29$ \\
\hline Pam50+Claudin-low subtype & | Basal | claudin-low | | Her2 | LumA | LumB |NC | Normal \\
\hline Neoplasm histologic grade & 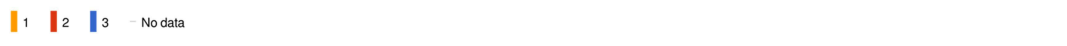 \\
\hline Tumor stage & 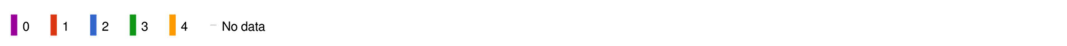 \\
\hline Lymph nodes examined positive & 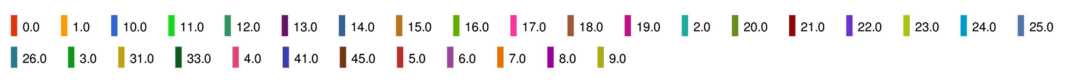 \\
\hline Cancer type detailed & $\begin{array}{l}\text { | Breast | Breast Invasive Ductal Carcinoma } \| \text { Breast Invasive Lobular Carcinoma || Breast Invasive Mixed Mucinous Carcinoma || Breast Mixed Ductal and Lobular Carcinoma } \\
\text { | Metaplastic Breast Cancer } \quad-\text { No data }\end{array}$ \\
\hline Tumor other histologic subtype & | DuctalNST | Lobular | Medullary | Metaplastic | Mixed | Mucinous $\mid$ Other | Tubular/cribritorm - No data \\
\hline BX1 mRNA expression & $3 \square$ \\
\hline
\end{tabular}

B

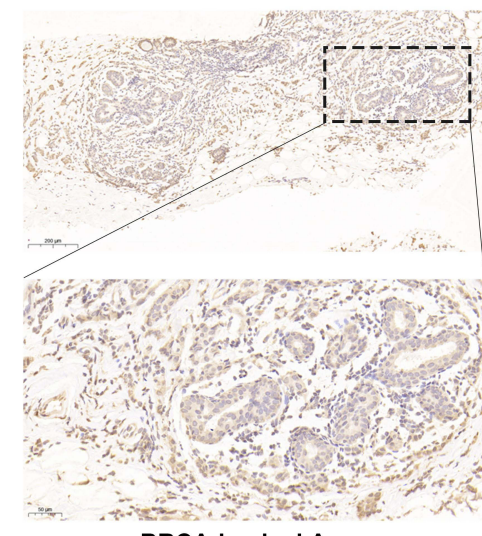

BRCA-luminal A

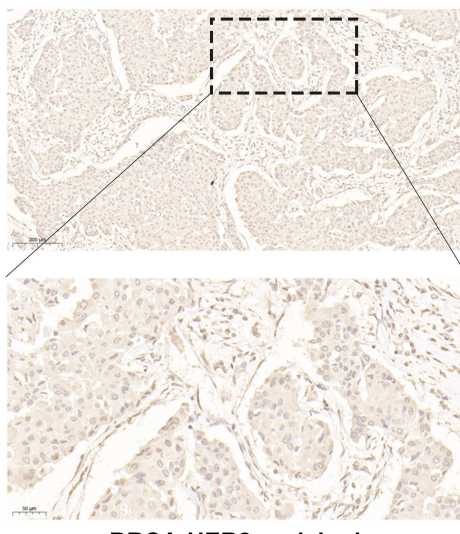

BRCA-HER2-enriched

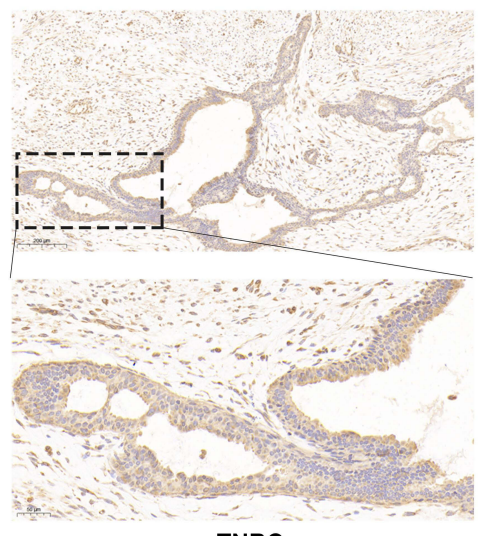

TNBC
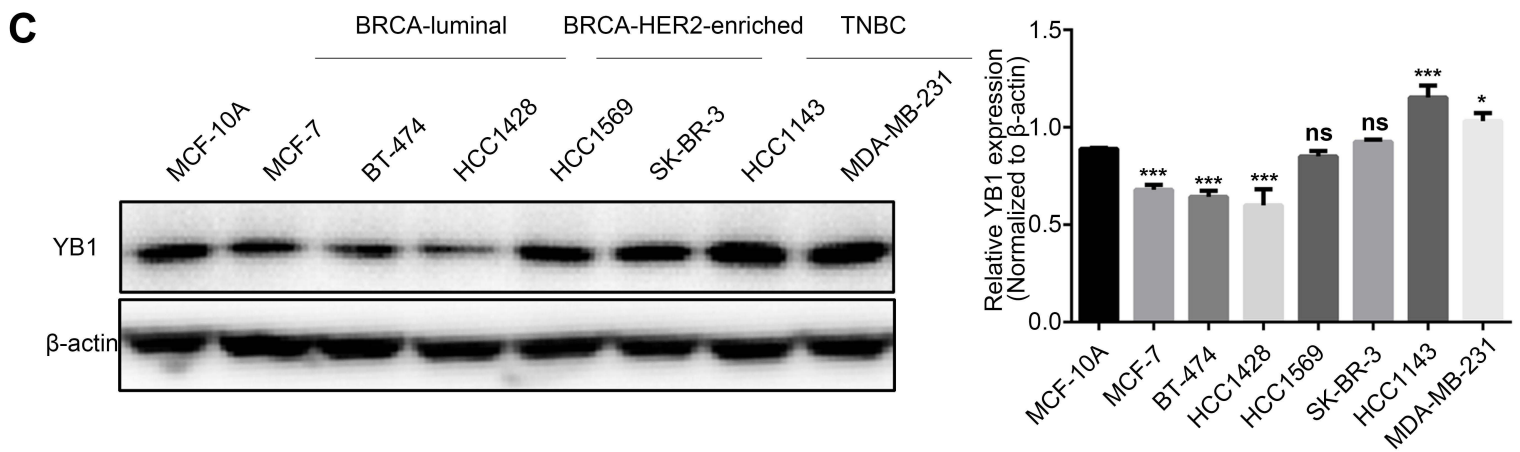

Figure 3 The correlation between YBXI expression and clinicopathological parameters in BRCA.

Notes: (A) The clustering of clinicopathological parameters according to the YBXI mRNA level using cBioPeortal database. (B) Immunohistochemical staining of YBI in $B R C A$ samples with different molecular subtypes. $(\mathbf{C})$ The different protein expression of $Y B I$ in human breast epithelial cells and aseries of $B R C A$ cell lines. $* P<0.05$ and $* * * P<0.00$ I vs control group.

Abbreviations: BRCA, breast cancer; cBioPortal, the cBio Cancer Genomics Portal database; ns, not significant; TNBC, triple-negative breast cancer; YBXI, Y-box binding protein I. 
A

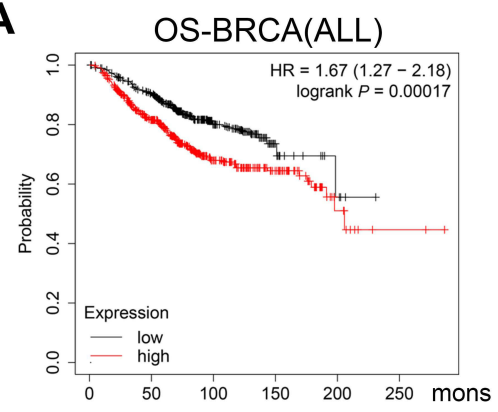

OS-BRCA(luminaA)

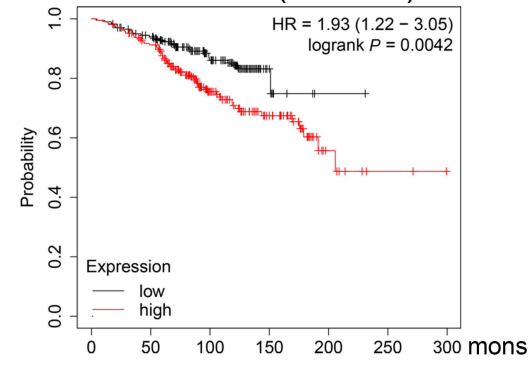

OS-BRCA(luminaB)

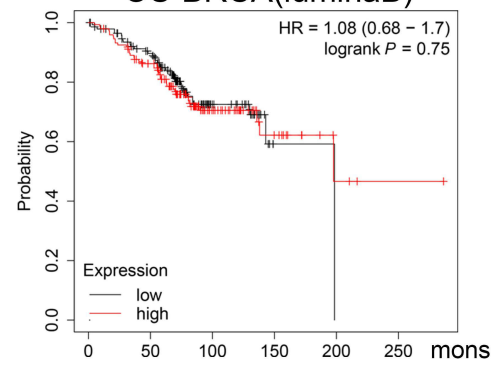

OS-BRCA(HER2-enriched)
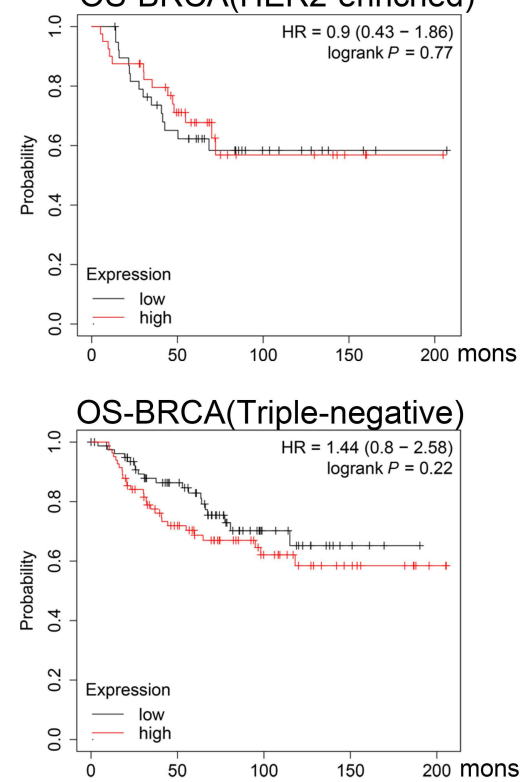

B
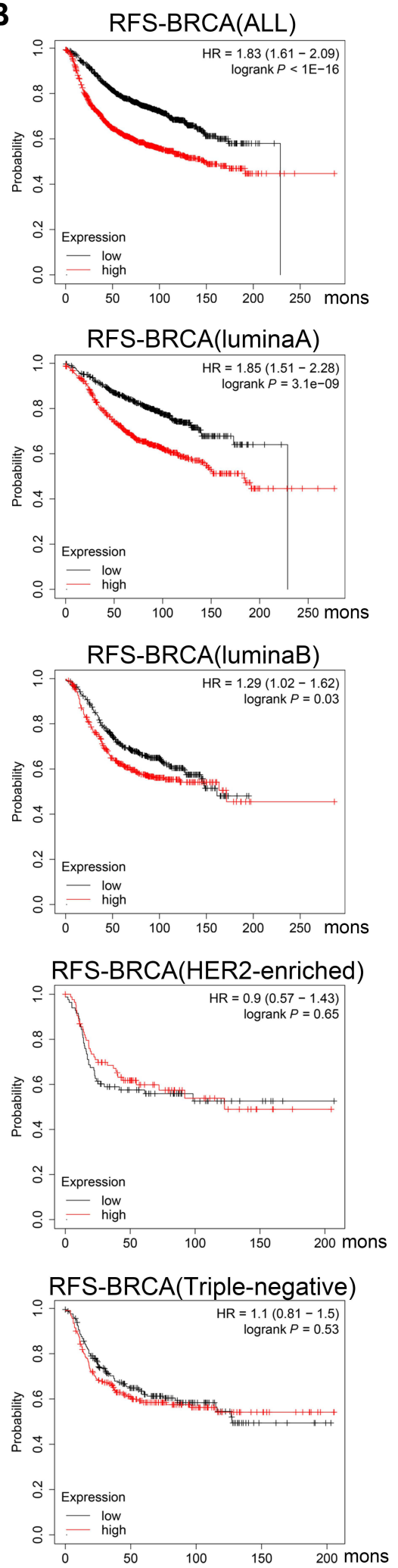

Figure 4 Survival curves comparing the high and low expression of $Y B X I$ in BRCA by Kaplan-Meier plotter database.

Notes: (A) Survival curves of OS in the entire BRCA cohort and different subtypes; (B) Survival curves of RFS in the entire BRCA cohort and different subtypes. Abbreviations: BRCA, breast cancer; HR, hazard ratio; OS, overall survival; RFS, relapse-free survival; YBXI, Y-box binding protein I. 
Table I Correlation of YBXI Expression and Clinical Prognosis in Breast Cancer with Different Clinicopathological Parameters by Kaplan-Meier Plotter

\begin{tabular}{|c|c|c|c|c|c|c|}
\hline \multirow[t]{2}{*}{ Clinicopathological Characteristics } & \multicolumn{3}{|c|}{ Overall Survival $(n=\mid 402)$} & \multicolumn{3}{|c|}{ Relapse-Free Survival (n=3955) } \\
\hline & $\mathbf{N}$. & Hazard Ratio & $P$-value & $\mathbf{N}$. & Hazard Ratio & $P$-value \\
\hline \multicolumn{7}{|l|}{ ER STATUS } \\
\hline Positive & 548 & $1.27(0.88-1.81)$ & 0.20 & 2061 & $1.24(1.06-1.47)$ & 0.0088 \\
\hline Negative & 251 & I.II (0.7-I.75) & 0.66 & 801 & $0.85(0.68-1.07)$ & 0.17 \\
\hline \multicolumn{7}{|l|}{ HER2 STATUS } \\
\hline Positive & 129 & $0.73(0.36-1.49)$ & 0.39 & 252 & $0.99(0.64-1.52)$ & 0.95 \\
\hline Negative & 130 & $1.34(0.55-3.26)$ & 0.52 & 800 & $I .84(I .4 I-2.4 I)$ & $6.4 \mathrm{e}-6$ \\
\hline \multicolumn{7}{|l|}{ INTRINSIC SUBTYPE } \\
\hline Luminal A & 410 & $1.93(1.22-3.05)$ & 0.0042 & 1295 & $\mathrm{I} .85(\mathrm{I} .5 \mathrm{I}-2.28)$ & $3.1 \mathrm{e}-9$ \\
\hline Luminal B & 290 & $1.08(0.68-1.70)$ & 0.75 & 770 & $1.29(1.02-1.62)$ & 0.03 \\
\hline HER2-enriched & 79 & $0.90(0.43-1.86)$ & 0.77 & 168 & $0.90(0.57-1.43)$ & 0.65 \\
\hline Basal-like & 162 & I.44(0.80-2.58) & 0.22 & 414 & $1.10(0.81-1.50)$ & 0.53 \\
\hline \multicolumn{7}{|l|}{ LYMPH NODE STATUS } \\
\hline Positive & 313 & $1.05(0.7|-| .55)$ & 0.80 & 1133 & $1.49(1.23-1.82)$ & $6.1 e-5$ \\
\hline Negative & 594 & $1.79(1.22-2.62)$ & 0.0025 & 2020 & $1.44(1.21-1.70)$ & $2.4 \mathrm{e}-5$ \\
\hline
\end{tabular}

Abbreviations: ER, estrogen receptor; HER2, human epidermal growth factor receptor 2; N, number of patients; YBXI, Y-box binding protein I.

patients, we found a synergy of high $Y B X 1$ expression with high M2 infiltration which yielded the lowest survival outcome (Figure 6B). In the 3D macrophage-tumor cell coculture system, although $Y B X 1$ knockdown could cause the inhibition of cell viability when BRCA cells were cultured alone, the addition of macrophages remarkably aggravated the inhibitory effect (Figure 7A and B). However, the phenomenon exist only in the luminal BRCA cells MCF-7 and BT-474, but not in TNBC cells MDA-MB-231 (Figure 7B). All these results indicate that $Y B X 1$ overexpression accelerates the luminal BRCA progression by promoting macrophage polarization from M1 to M2.

\section{The Positive Correlation Between YBXI and $\mathrm{T}$ Cell Exhaustion in BRCA}

In addition to markers of tumor associated macrophages, gene markers of $\mathrm{T}$ cell exhaustion were demonstrated to correlate significantly with $Y B X 1$ expression as well (BRCA: IDO1: rs=0.388, $P=4.93 \mathrm{e}-37 ; P D C D 1$ : $\mathrm{rs}=0.249$, $P=1.52 \mathrm{e}-15 ; \quad C T L A 4: \quad$ rs $=0.321, \quad P=2.54 \mathrm{e}-25 ; \quad L A G 3$ : $\mathrm{rs}=0.294, P=2.55 \mathrm{e}-21 ;$ TIM3: $\mathrm{rs}=0.169, P=8.62 \mathrm{e}-8$; luminal A subtype: $I D O 1$ : rs $=0.193, P=1.02 \mathrm{e}-5 ; P D C D 1: \mathrm{rs}=0.147$, $P=7.89 \mathrm{e}-4 ; C T L A 4: \mathrm{rs}=0.146, P=8.99 \mathrm{e}-4 ; L A G 3: \mathrm{rs}=0.070$, $P=1.10 \mathrm{e}-1 ; \quad$ TIM3: $\quad \mathrm{rs}=0.275, \quad P=1.99 \mathrm{e}-10 ; \quad$ luminal
B subtype: IDO1: $\quad$ rs $=0.224, \quad P=1.78 \mathrm{e}-3 ; \quad P D C D 1$ : rs $=0.187, P=9.51 \mathrm{e}-3 ; C T L A 4:$ rs $=0.163, P=2.37 \mathrm{e}-2 ; L A G 3$ : $\mathrm{rs}=0.158, P=2.84 \mathrm{e}-2$; TIM3: $\mathrm{rs}=0.186, P=9.73 \mathrm{e}-3$; Table 2). Analysis from cBioPortal gave the similar correlation of $Y B X 1$ with IDO1, PDCD1, CTLA4 and LAG3 (Figure 6D). Since IDOI and CTLA4 showed the strongest correlations with $Y B X 1$, immunohistochemical staining of IDO1, CTLA4 and YB1 was performed in luminal BRCA samples, and significant decreases of IDO1 and CTLA4 expression were demonstrated along with the downregulation of YB1 expression (Figure 6E). Additionally, scatter plot from LinkedOmics showed the significant positive association between $Y B X 1$ and some other immunosuppression-related genes such as CTPS, LYAR etc (Figure 6C). ${ }^{38,39}$

To confirm the positive correlation between $Y B X 1$ and $T$ cell suppression, a co-culture system was used to test the effect of $Y B X 1$ knockdown in BRCA cells on the functions of activated $\mathrm{CD} 8+\mathrm{T}$ cells. $Y B X 1$ depletion resulted in significant increases in LDH release and secretion of IL2 and IFN- $\gamma$ in co-cultures of T cells with MCF-7 or BT474 luminal BRCA cells but not MDA-MB-231 TNBC cells (Figure $7 \mathrm{C}-\mathrm{E}$ ). These results indicate that $Y B X 1$ overexpression in luminal BRCA induces $\mathrm{T}$ cell exhaustion thereby accelerating tumor progression. 


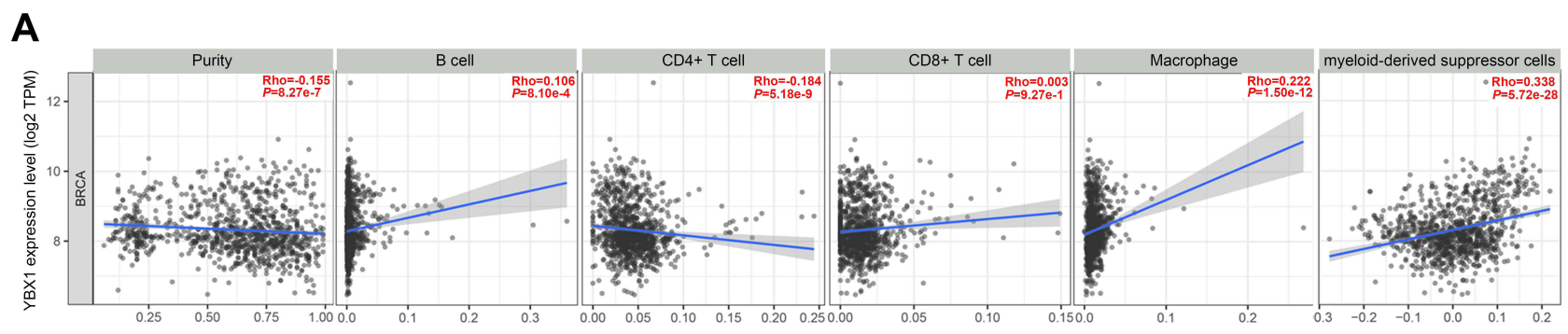

B

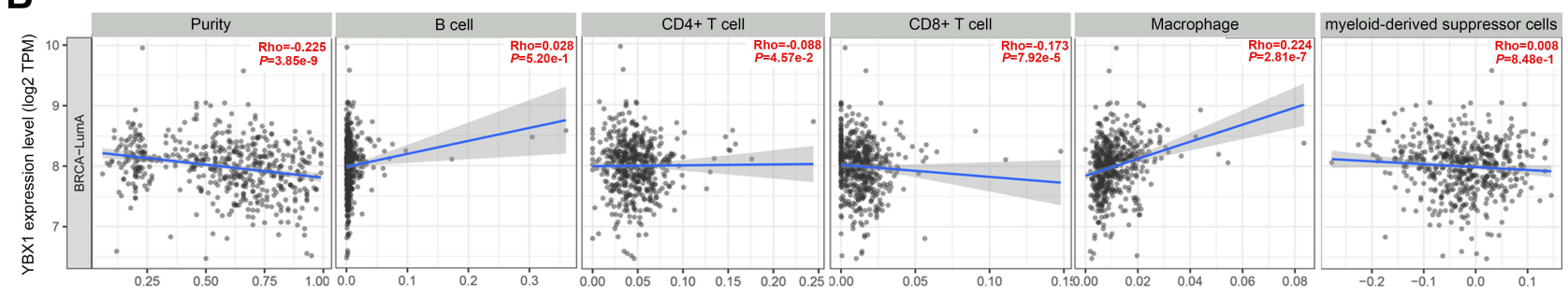

C
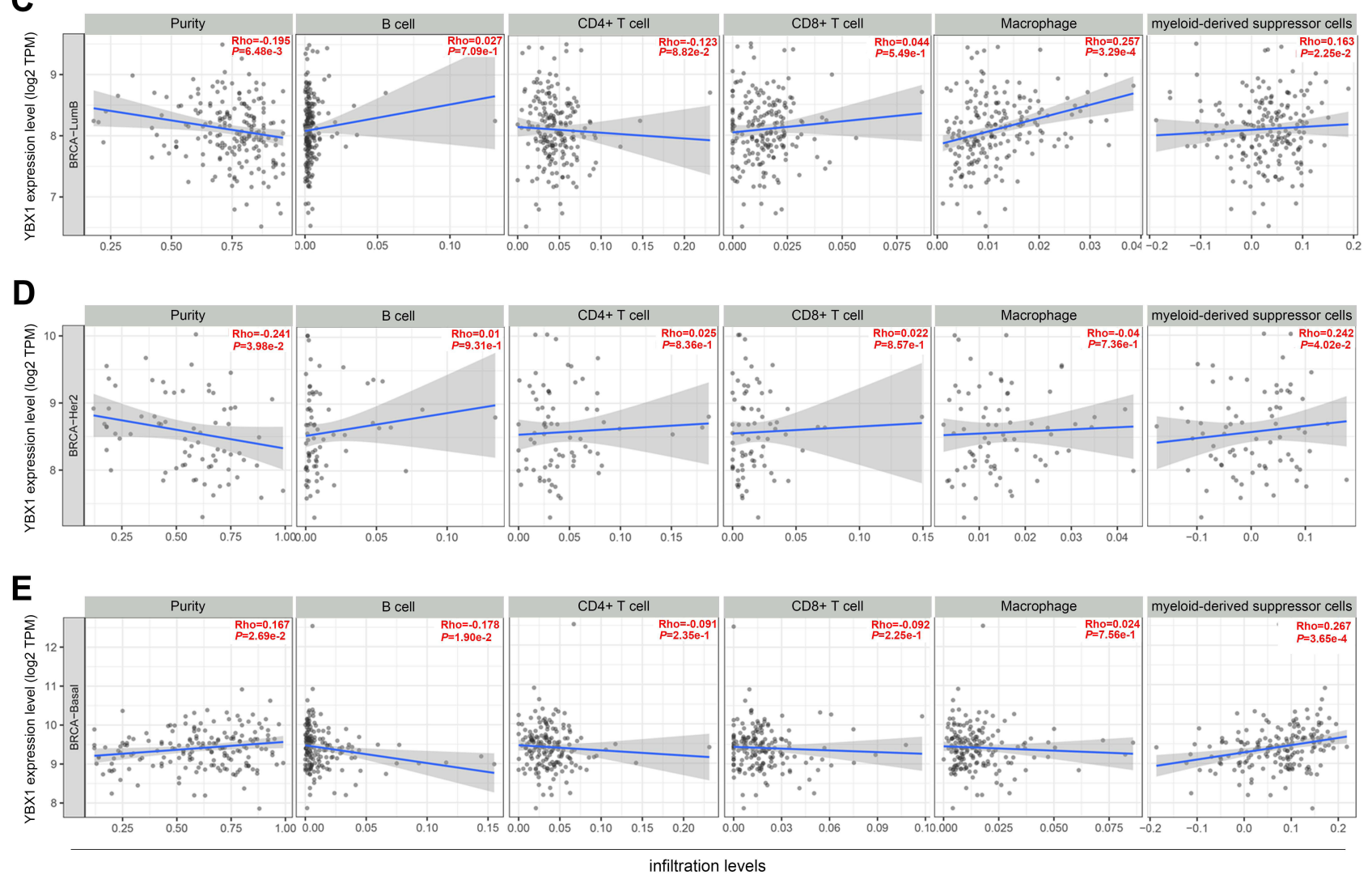

Figure 5 Correlation of YBXI expression with immune cell infiltration level in (A) the entire BRCA cohort; (B) the luminal A subtype; (C) the luminal B subtype; (D) the HER2-enriched subtype; and (E) the triple-negative subtype by TIMER database.

Abbreviations: BRCA, breast cancer; TIMER, the Tumor Immune Estimation Resource database; YBXI, Y-box binding protein I.

\section{Discussion}

$Y B X 1$ is known as an oncogene that facilitates malignant progression and induces chemoresistance in many cancers. ${ }^{40}$ In this study, on the basis of studying the expression pattern of $Y B X 1$ in different BRCA subtypes, we further investigated the impact of immune cell infiltration along with $Y B X 1$ overexpression on the prognosis of BRCA. We found that high YBXI expression predicted significantly shorter survival in BRCA, especially the luminal subtypes. Moreover, this correlation was 


\begin{tabular}{|c|c|c|c|c|c|c|c|c|c|}
\hline & \multirow{2}{*}{ 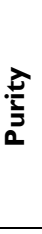 } & 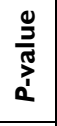 & 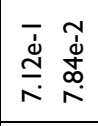 & 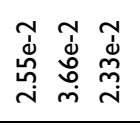 & 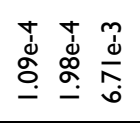 & 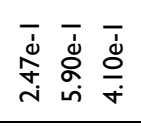 & 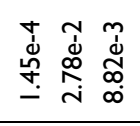 & $\begin{array}{l}\stackrel{m}{d} \\
\stackrel{\infty}{=} \\
\stackrel{-}{=}\end{array}$ & 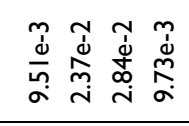 \\
\hline & & ù & $\underset{0}{0} \frac{\overline{0}}{0}$ & $\frac{\bar{\sigma}}{0} \frac{\bar{n}}{0} \frac{t}{0}$ & 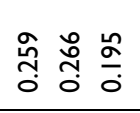 & 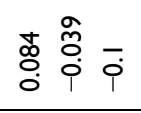 & $\stackrel{\bar{\lambda}}{0} \frac{0}{0} \frac{0}{0}$ & 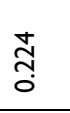 & $\frac{\hat{0}}{0} \frac{m}{0} \frac{\stackrel{\infty}{\circ}}{0} \frac{\infty}{0}$ \\
\hline & \multirow{2}{*}{ 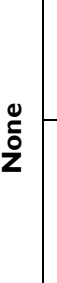 } & 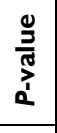 & 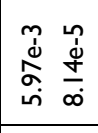 & 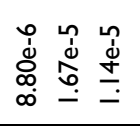 & 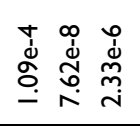 & 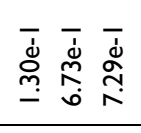 & 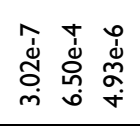 & 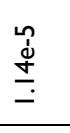 & 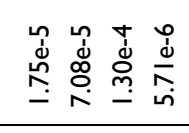 \\
\hline & & ò & 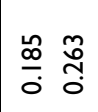 & 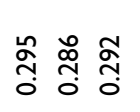 & ڤ્ণ & 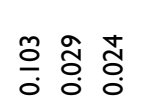 & 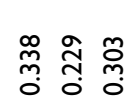 & స̃ స్ & 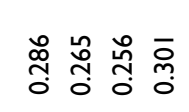 \\
\hline \multirow{4}{*}{ 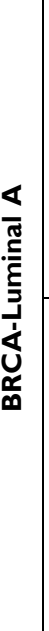 } & \multirow{2}{*}{ 氦 } & 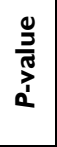 & 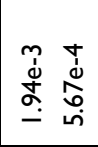 & 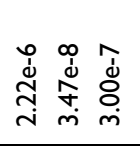 & 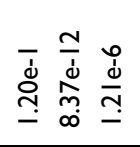 & 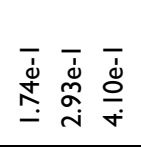 & 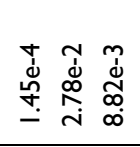 & 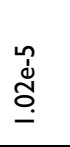 & 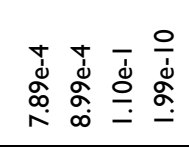 \\
\hline & & نे & $\frac{\stackrel{\circ}{\circ}}{0} \frac{\bar{n}}{0}$ & 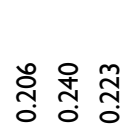 & 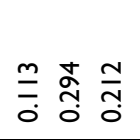 & 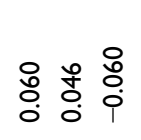 & సิํㅇㅇํㅇ & $\frac{a}{0}$ & 新劣 \\
\hline & \multirow{2}{*}{$\stackrel{0}{0}$} & 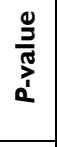 & 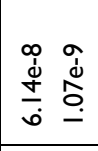 & 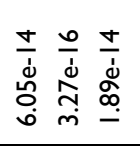 & 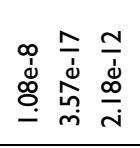 & 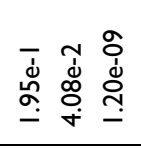 & 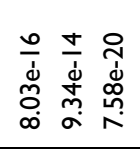 & $\begin{array}{l}\overline{\bar{d}} \\
\dot{\alpha} \\
\stackrel{\alpha}{\dot{j}}\end{array}$ & 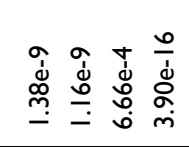 \\
\hline & & نे & 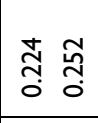 & 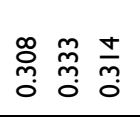 & 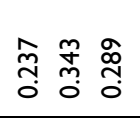 & 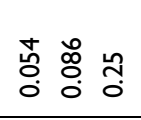 & $\stackrel{m}{m} \overline{0}$ m & స̂. & 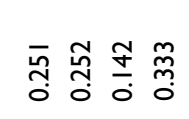 \\
\hline \multirow{4}{*}{ zot } & \multirow{2}{*}{ 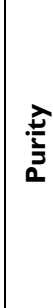 } & 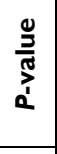 & 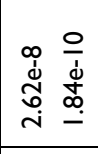 & 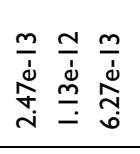 & 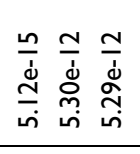 & 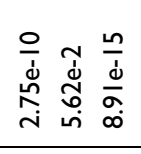 & 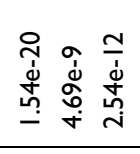 & $\begin{array}{l}\hat{m} \\
\dot{d} \\
\tilde{m} \\
\sigma \\
\dot{\sigma}\end{array}$ & 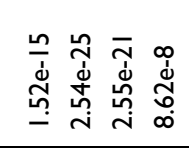 \\
\hline & & نे & ๓ & 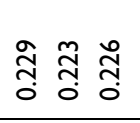 & 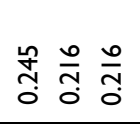 & 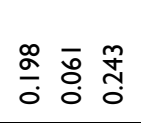 & 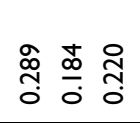 & $\underset{\substack{\infty \\
\hdashline}}{0}$ & 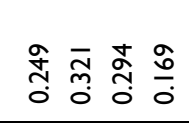 \\
\hline & \multirow{2}{*}{ ๕̊ } & 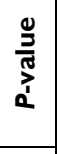 & 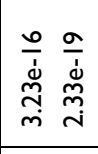 & 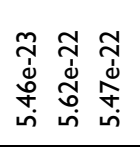 & 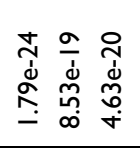 & 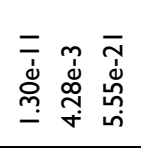 & 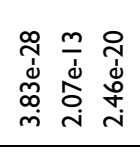 & 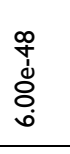 & 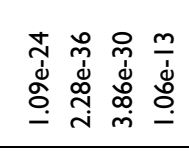 \\
\hline & & نे & స્ડ & 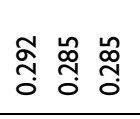 & 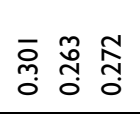 & 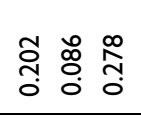 & 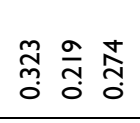 & $\frac{a}{\sigma}$ & 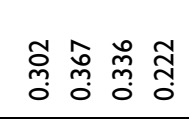 \\
\hline \multicolumn{3}{|c|}{ 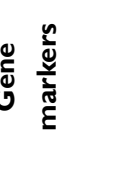 } & 造 & 岂 û̃ & ㄱํ & 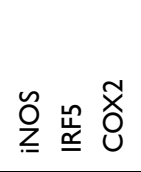 & 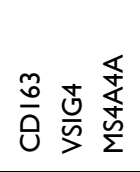 & $\begin{array}{l}\overline{0} \\
\underline{0}\end{array}$ & 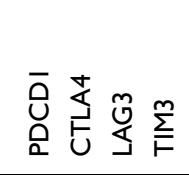 \\
\hline \multicolumn{3}{|l|}{ 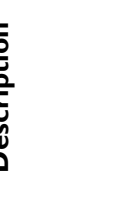 } & $\overline{\overline{\mathrm{J}}}$ & 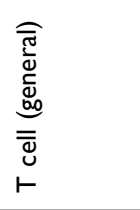 & $\sum_{\leqslant}$ & 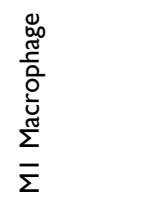 & 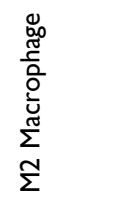 & $\overline{\bar{\Phi}}$ & \\
\hline
\end{tabular}


A

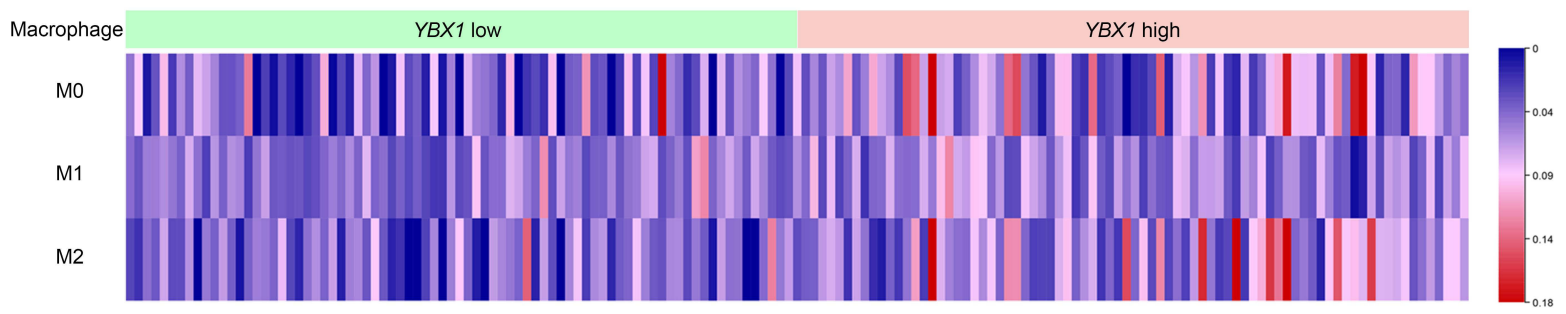

B

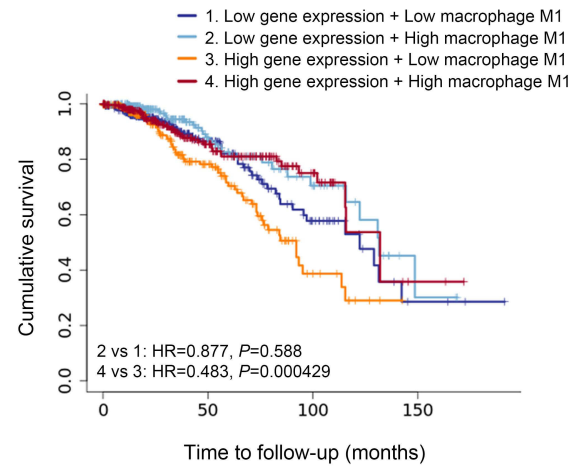

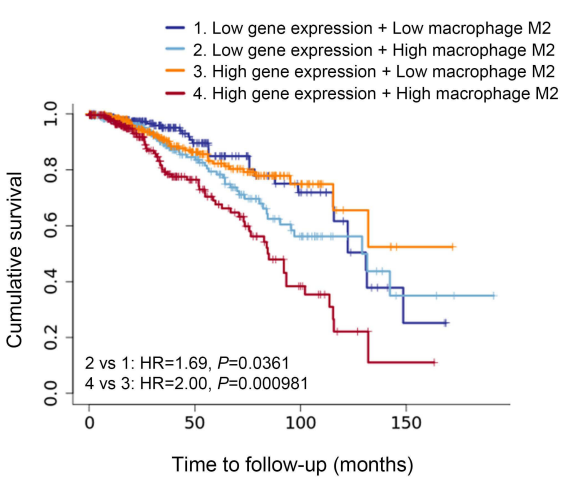

C

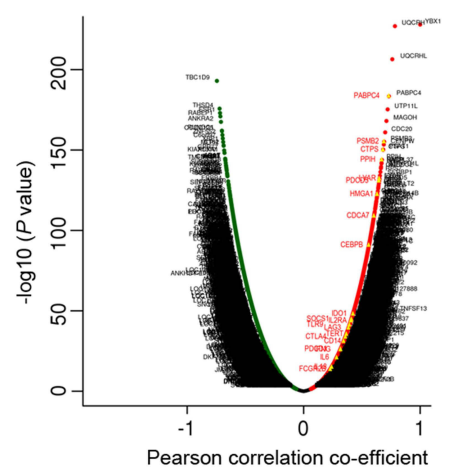

D
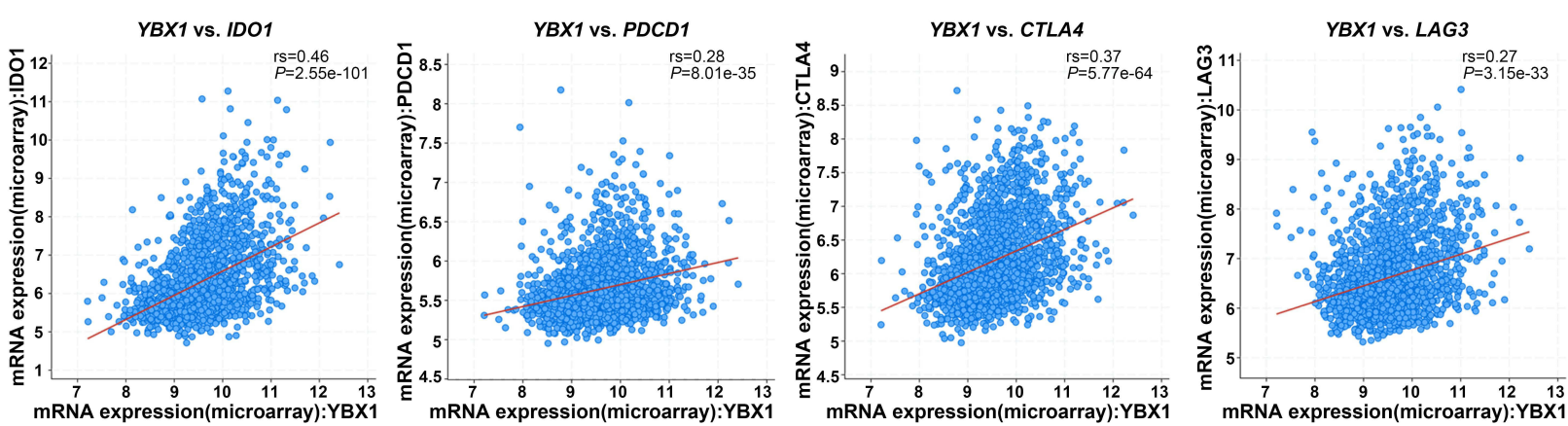

E
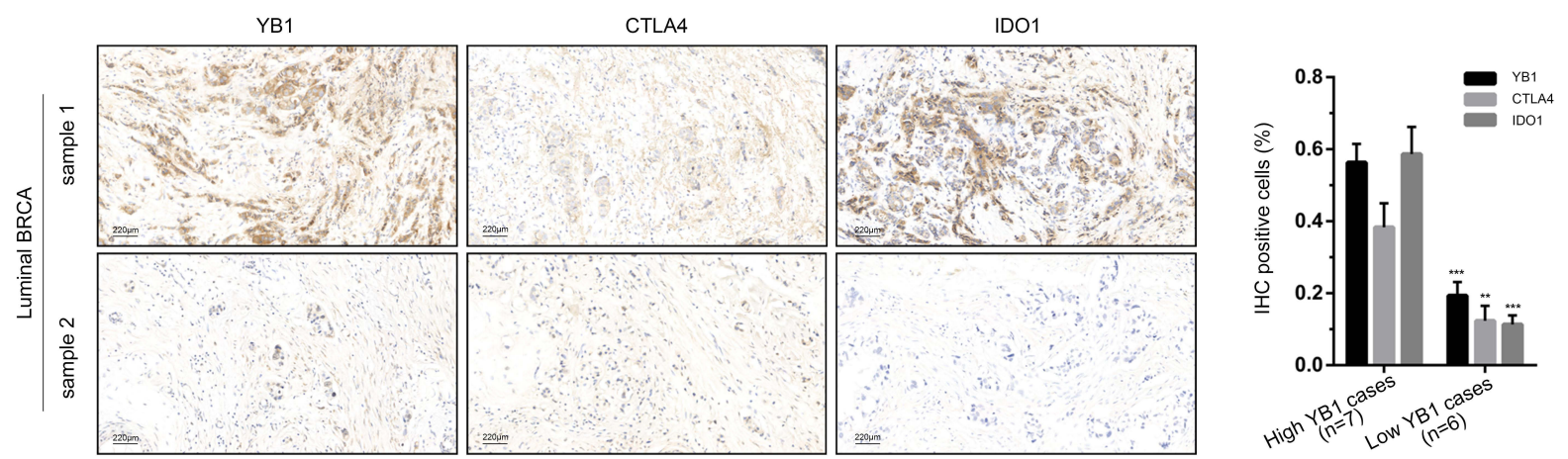

Figure 6 Verification of the correlation between $Y B X I$ and immune cell infiltration in BRCA.

Notes: (A) Infiltration levels of different type of macrophages in samples from GSEI456 dataset with different YBXI expression level. (B) Kaplan-Meier curves from the TIMER database showing the impacts of $Y B X I$ expression and MI/M2 infiltration on survival of BRCA patients. (C) Ascatter plot of correlation between $Y B X I$ and various genes especially those associated with immunosuppression (annotated in red) in BRCA. (D) Correlation of YBXI with IDOI, PDCDI, CTLA4 and LAG3 in BRCA verified by cBioPortal database. (E) Immunohistochemical staining of IDOI and CTLA4 in luminal BRCA samples with different $Y B I$ expression levels. $* * P<0.01$ and $* * * P<0.00 I$ vs corresponding YBI high-expressing groups.

Abbreviations: BRCA, breast cancer; cBioPortal, the cBio Cancer Genomics Portal database; CTLA4, cytotoxic T-lymphocyte associated protein 4; IDOI, indoleamine 2,3-dioxygenase I; LAG3, lymphocyte activating 3; NES, normalized enrichment score; HR, hazard ratio; PDCDI programmed cell death I; TIMER, Tumor Immune Estimation Resource; TCGA, The Cancer Genome Atlas; YBXI, Y-box binding protein I. 
A

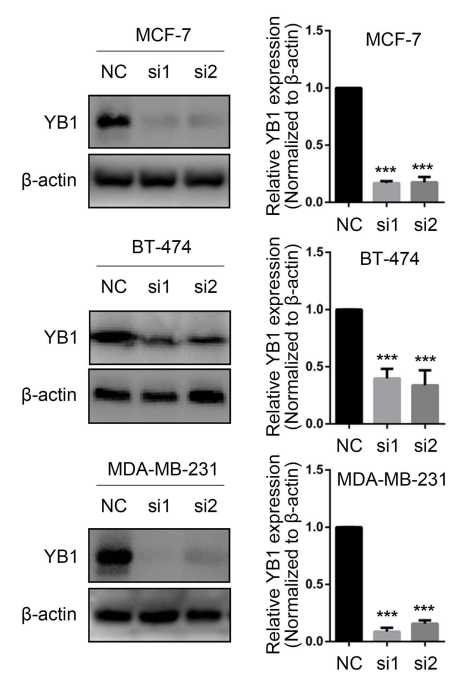

B
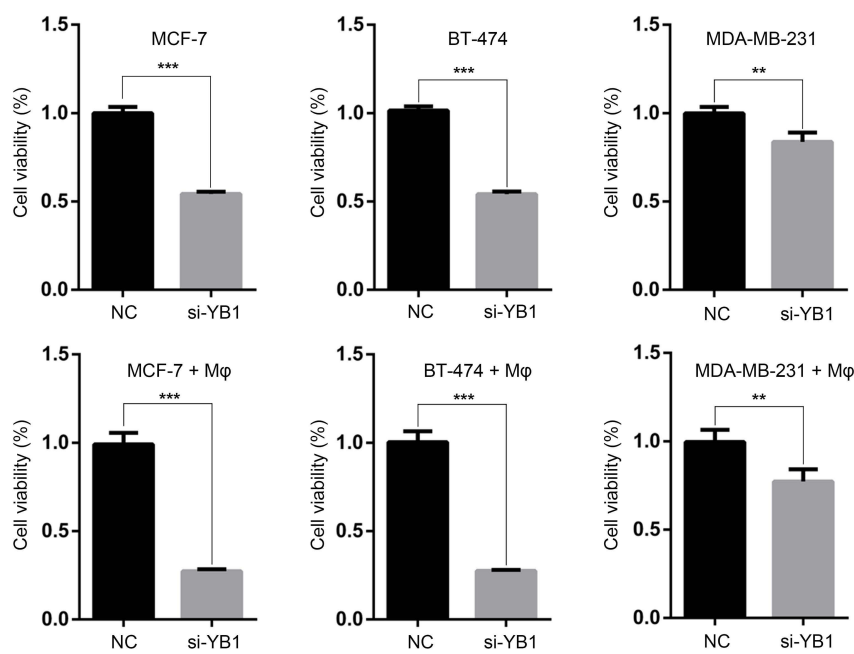

C
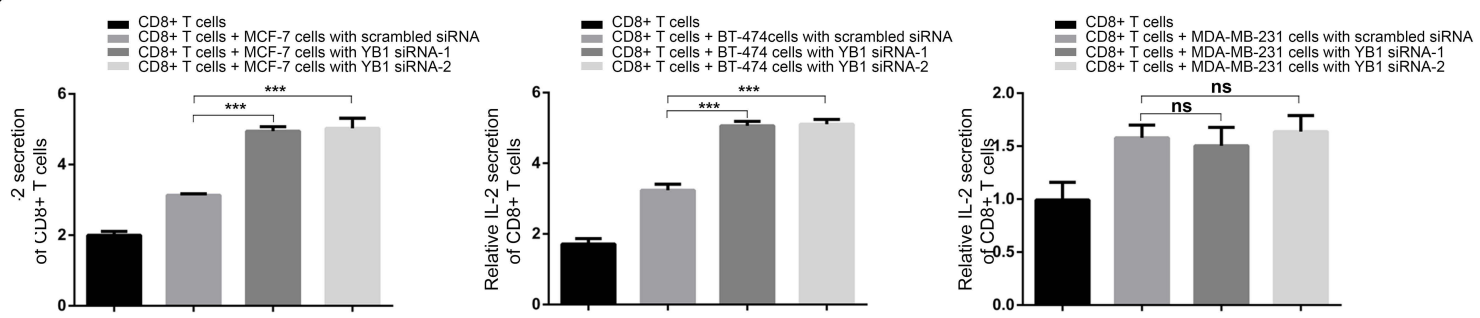

D
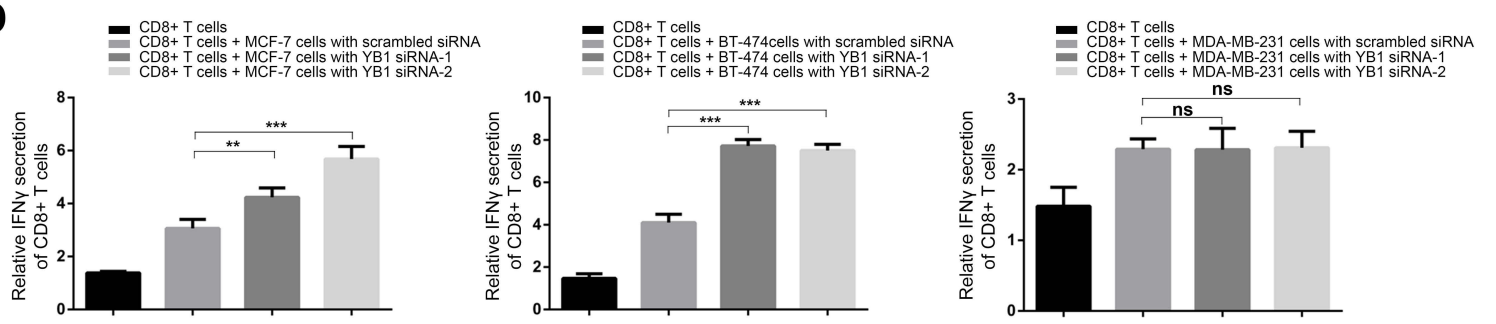

E
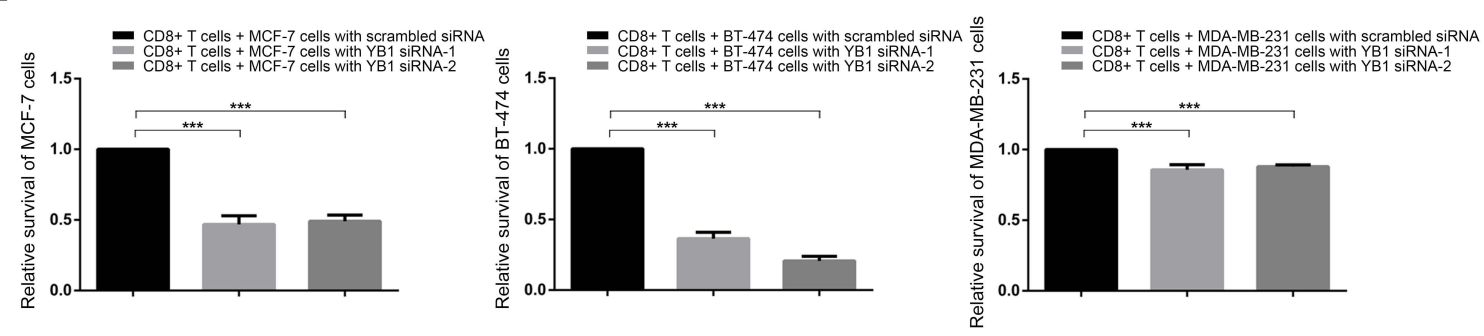

Figure 7 Effect of $Y B X I$ knockdown on the cytotoxicity of macrophages and CD8+ T cells in co-culture system of BRCA cells and immunocytes.

Notes: (A) YBXI was effectively knocked down by siRNAs in MCF-7, BT-474, and MDA-MB-23I cells. (B) BRCA cells with or without knockdown of YBXI were cultured alone or co-cultured with peritoneal macrophages, and cell viability was evaluated. For luminal BRCA cells, namely MCF-7 and BT-474, the decrease of cell viability after YBXI knockdown were more dramatic in co-cultures compared with the single culture system. However, no difference was observed for MDA-MB-23I TNBC cells. (C) IL-2 production by activated CD8+ Tcells co-cultured with BRCA cells transfected with YBXI siRNA or scrambled negative control siRNA. YBXI knockdown significantly increased IL-2 secretion in co-cultures of CD8+ Tcells with MCF-7 or BT-474 cells but not in the co-culture with MDA-MB-23I cells. (D) IFN- $\gamma$ production by activated CD8 + Tcells co-cultured with BRCA cells transfected with YBXI or scrambled negative control siRNA. YBXI knockdown significantly increased IFN- $\gamma$ secretion in the cocultures of CD8+ Tcells and MCF-7 or BT-474 cells but not in the co-culture with MDA-MB-23I cells. (E) The relative survival of BRCA cells represented by the release of $\mathrm{LDH}$ in co-cultures of CD8+ Tcells and BRCA cells with or without YBXI knockdown. YBXI depletion markedly reduced the survival of MCF-7 and BT-474 cells but not MDA-MB-23I cells. ${ }^{*} * P<0.01$, $* * * P<0.001$ compared with corresponding negative control groups.

Abbreviations: BRCA, breast cancer; IFN- $\gamma$, interferon- $\gamma$; IL-2, interleukin-2; LDH, lactate dehydrogenase; NC, negative control; YBXI, Y-box binding protein I. 
demonstrated to be associated with M2 macrophage infiltration and $\mathrm{T}$ cell exhaustion.

Several studies have reported an overall decrease in $Y B X 1$ expression in BRCA. ${ }^{41-43}$ Our study confirms it and further reveals the differential expression of $Y B X 1$ in the four molecular subtypes. $Y B X 1$ was downregulated in the luminal BRCA subtypes but upregulated in TNBC compared to normal tissues. A possible explanation for the opposite patterns is that $Y B X 1$ is negatively correlated with ER and progesterone receptor (PR) levels. ${ }^{8,44-47}$ Kuwano et al summarized seventy independent studies involving $Y B X 1$ expression in cancers and demonstrated positive correlation of $Y B X I$ with common oncogenes such as epidermal growth factor receptor (EGFR), androgen receptor $(A R)$, and ATP binding cassette subfamily B member $1(A B C B 1)$, but a strong negative correlation with ER $\alpha$ in BRCA. ${ }^{8}$ Bansal et al assessed the expression of ER, PR and YB1 by immunohistochemistry in the specimens of seventy-four BRCA patients, finding significantly negative correlation of YB1 with ER and PR expression. ${ }^{46}$ Since hormone receptors, ER and PR, signify luminal BRCA rather than TNBC, $Y B X 1$ is more prone to be expressed highly in TNBC rather than luminal BRCA.

As for prognosis, the present study found that high expression of $Y B X 1$ predicted worse clinical outcomes in BRCA in general but especially in the luminal subtypes. However, a retrospective study that analyzed the clinicopathologic characteristics of 4049 BRCA cases showed that $Y B X 1$ overexpression was linked to shorter RFS and disease-specific survival across all BRCA subtypes including HER2-positive BRCA and TNBC. ${ }^{48}$ This inconsistency may be explained by methodological differences in the detection of $Y B X 1$ expression. Our study detected the mRNA level of $Y B X 1$ from GEO, EGA, and TCGA using the JetSet best probe set 208628_s_at, whereas the previous study assessed the protein level of $Y B X 1$ from a large tumor tissue microarray by immunohistochemistry. The incomplete correlation between the expression profiles of $Y B X 1 \mathrm{mRNA}$ and protein could underlie the observed discrepancy in clinical outcomes. ${ }^{49,50}$ Nonetheless, the conclusion can be drawn that, for luminal BRCA, the subtypes with positive expression of hormone receptors and less frequency of $Y B X 1$ expression, high $Y B X 1$ expression can be identified as a predictive biomarker of poor survival.

Given the downregulation and the prognostic value of $Y B X 1$ expression in luminal BRCA, we can infer that even if not participating in tumorigenesis, $Y B X 1$ overexpression is involved in the progression of luminal BRCA. As tumor microenvironment alteration is a vital factor in tumor progression, ${ }^{51}$ we explored whether the worse prognosis of $Y B X 1$ high-expressing groups resulted from immune cell infiltration into the tumor microenvironment. Our analysis of public datasets along with results from the immunocyte-tumor cell co-culture assay revealed the positive correlation between $Y B X 1$ and M2 macrophage infiltration and $\mathrm{T}$ cell exhaustion in BRCA. M2 macrophages exhibit a protumoral phenotype and $\mathrm{T}$ cell exhaustion leads to immune escape of cancer cells, which together facilitate tumor progression including relapse in situ and distant metastasis. ${ }^{52,53}$ Interestingly, consistent with the prognostic results, the significant correlation of $Y B X 1$ with $\mathrm{M} 2$ infiltration and $\mathrm{T}$ cell exhaustion was only observed in luminal BRCA rather than other subtypes, implying that these immune mechanisms contributed to the reduced survival of patients with high $Y B X 1$ expression. Recent investigations have provided some potential mechanisms about the impact of $Y B X 1$ on macrophage activities. ${ }^{54,55}$ For example, Hessman et al evaluated the effect of YB1 on the tumor necrosis factor alpha (TNF $\alpha)$-TNF receptor interaction in macrophages with the flow cytometric TNF $\alpha$ binding assay, demonstrating that YB1 competed with $\mathrm{TNF} \alpha$ for receptor binding and thereby inhibited the TNF $\alpha$ signaling cascade that induces macrophage polarization from M2 to M1; $;^{54}$ this was supported by our finding that $Y B X 1$ promoted macrophage polarization from M1 to M2. For another thing since luminal BRCA with high $Y B X 1$ expression suffered from greater $\mathrm{T}$ cell exhaustion and presented higher levels of IDOI and CTLA4, IDOI/ CTLA4 inhibitors, namely ipilimumab and indoximod, may bring more clinical benefits for this subgroup. Moreover, the addition of M1 polarization agents, namely IFN- $\gamma$ and lipopolysaccharide, was suggested to augment the treatment efficacy. Thus, a promising therapeutic strategy for $Y B X 1$ high-expressing luminal BRCA may be hidden in the combination of surgery, chemotherapy drugs, antiestrogens, IDO1/CTLA4 inhibitors and M1 polarization agents, which warrants evaluation in clinical trials.

Our study has several limitations. Firstly, most of the data analyzed in this study were obtained from the online databases. As for our own clinical cases, the lack of systematic follow-up made it difficult to accurately infer prognosis. Secondly, although statistically significant, the correlation between $Y B X 1$ and the markers of $\mathrm{T}$ cell 
exhaustion were not strong; therefore, large-sample studies with the expression data for $Y B X 1, I D O 1$ and CTLA4 are needed to verify the practical significance of this finding. Finally, our study lacked in vivo experimental data. Even a 3D co-culture system with immunocytes cannot replace animal models, which should be included in any future investigations.

In conclusion, high mRNA level of $Y B X 1$ predicts worse prognosis in BRCA especially with luminal subtypes, which may be associated with M2 macrophage infiltration and $\mathrm{T}$ cell exhaustion. These findings enlighten the combination of classic therapeutics with immune checkpoint inhibitors and M1 polarization agents for $Y B X 1$ high-expressing luminal BRCA patients in the future trials.

\section{Acknowledgment}

The authors thank the members of the Department of Pathology, Shandong Cancer Hospital and Institute for the helpful pathological sectioning.

\section{Disclosure}

The authors declare no conflicts of interest for this work.

\section{References}

1. Siegel RL, Miller KD, Jemal A. Cancer statistics, 2019. CA Cancer J Clin. 2019;69(1):7-34.

2. Wu CY, Du SL, Zhang J, Liang AL, Liu YJ. Exosomes and breast cancer: a comprehensive review of novel therapeutic strategies from diagnosis to treatment. Cancer Gene Ther. 2017;24(1):6-12. doi: $10.1038 /$ cgt.2016.69

3. Engstrom MJ, Opdahl S, Hagen AI, et al. Molecular subtypes, histopathological grade and survival in a historic cohort of breast cancer patients. Breast Cancer Res Treat. 2013;140(3):463-473. doi:10.1007/ s10549-013-2647-2

4. Gouri A, Dekaken A, El Bairi K, et al. Plasminogen activator system and breast cancer: potential role in therapy decision making and precision medicine. Biomark Insights. 2016;11:105-111. doi:10.4137/ BMI.S33372

5. Li X, Yang J, Peng L, et al. Triple-negative breast cancer has worse overall survival and cause-specific survival than non-triple-negative breast cancer. Breast Cancer Res Treat. 2017;161(2):279-287. doi:10.1007/s10549-016-4059-6

6. Smerage JB, Barlow WE, Hortobagyi GN, et al. Circulating tumor cells and response to chemotherapy in metastatic breast cancer: SWOG S0500. J clin oncol. 2014;32(31):3483-3489. doi:10.1200/ JCO.2014.56.2561

7. Chen B, Lai J, Dai D, Chen R, Li X, Liao N. JAK1 as a prognostic marker and its correlation with immune infiltrates in breast cancer. Aging. 2019;11(23):11124-11135. doi:10.18632/aging.102514

8. Kuwano M, Shibata T, Watari K, Ono M. Oncogenic Y-box binding protein-1 as an effective therapeutic target in drug-resistant cancer. Cancer Sci. 2019;110(5):1536-1543. doi:10.1111/cas.14006

9. Kohno K, Izumi H, Uchiumi T, Ashizuka M, Kuwano M. The pleiotropic functions of the Y-box-binding protein, YB-1. BioEssays. 2003;25(7):691-698. doi:10.1002/bies.10300
10. Kretov DA, Clement MJ, Lambert G, et al. YB-1, an abundant core mRNA-binding protein, has the capacity to form an RNA nucleoprotein filament: a structural analysis. Nucleic Acids Res. 2019;47 (6):3127-3141. doi:10.1093/nar/gky1303

11. Woolley AG, Algie M, Samuel W, et al. Prognostic association of YB-1 expression in breast cancers: a matter of antibody. PLoS One. 2011;6(6):e20603. doi:10.1371/journal.pone.0020603

12. Cohen SB, Ma W, Valova VA, et al. Genotoxic stress-induced nuclear localization of oncoprotein YB-1 in the absence of proteolytic processing. Oncogene. 2010;29(3):403-410. doi:10.1038/ onc.2009.321

13. Lim JP, Shyamasundar S, Gunaratne J, Scully OJ, Matsumoto K, Bay BH. YBX1 gene silencing inhibits migratory and invasive potential via CORO1C in breast cancer in vitro. BMC Cancer. 2017;17 (1):201. doi:10.1186/s12885-017-3187-7

14. Maciejczyk A, Szelachowska J, Ekiert M, et al. Elevated nuclear YB1 expression is associated with poor survival of patients with early breast cancer. Anticancer Res. 2012;32(8):3177-3184.

15. Cho KH, Jeong BY, Park CG, Lee HY. The YB-1/EZH2/amphiregulin signaling axis mediates LPA-induced breast cancer cell invasion. Arch Pharm Res. 2019;42(6):519-530. doi:10.1007/s12272-01901149-6

16. Evdokimova V, Tognon $\mathrm{C}, \mathrm{Ng} \mathrm{T}$, et al. Translational activation of snail1 and other developmentally regulated transcription factors by YB-1 promotes an epithelial-mesenchymal transition. Cancer Cell. 2009;15(5):402-415. doi:10.1016/j.ccr.2009.03.017

17. Inoue I, Matsumoto K, Yu Y, Bay BH. Surmounting chemoresistance by targeting the Y-box binding protein-1. Anatomical Record. 2012;295(2):215-222. doi:10.1002/ar.22401

18. Liu SL, Sui YF, Lin MZ. MiR-375 is epigenetically downregulated due to promoter methylation and modulates multi-drug resistance in breast cancer cells via targeting YBX1. Eur Rev Med Pharmacol Sci. 2016;20(15):3223-3229.

19. Wang J, Djudjaj S, Gibbert L, et al. YB-1 orchestrates onset and resolution of renal inflammation via IL10 gene regulation. $J$ Cell Mol Med. 2017;21(12):3494-3505. doi:10.1111/jcmm.13260

20. Laman AG, Lathe R, Shepelyakovskaya AO, et al. Muramyl peptides activate innate immunity conjointly via YB1 and NOD2. Innate Immun. 2016;22(8):666-673. doi:10.1177/1753425916668982

21. Rhodes DR, Kalyana-Sundaram S, Mahavisno V, et al. Oncomine 3.0: genes, pathways, and networks in a collection of 18,000 cancer gene expression profiles. Neoplasia. 2007;9(2):166-180. doi:10.1593/neo.07112

22. Barretina J, Caponigro G, Stransky N, et al. The cancer cell line encyclopedia enables predictive modelling of anticancer drug sensitivity. Nature. 2012;483(7391):603-607. doi:10.1038/ nature 11003

23. Chandrashekar DS, Bashel B, Balasubramanya SAH, et al. UALCAN: a portal for facilitating tumor subgroup gene expression and survival analyses. Neoplasia. 2017;19(8):649-658. doi:10.1016/j. neo.2017.05.002

24. Cerami E, Gao J, Dogrusoz U, et al. The cBio cancer genomics portal: an open platform for exploring multidimensional cancer genomics data. Cancer Discov. 2012;2(5):401-404. doi:10.1158/21598290.CD-12-0095

25. Gao J, Aksoy BA, Dogrusoz U, et al. Integrative analysis of complex cancer genomics and clinical profiles using the cBioPortal. Sci Signal. 2013;6(269):pl1. doi:10.1126/scisignal.2004088

26. Gyorffy B, Lanczky A, Eklund AC, et al. An online survival analysis tool to rapidly assess the effect of 22,277 genes on breast cancer prognosis using microarray data of 1809 patients. Breast Cancer Res Treat. 2010;123(3):725-731. doi:10.1007/s10549-0090674-9

27. Li T, Fu J, Zeng Z, et al. TIMER2.0 for analysis of tumor-infiltrating immune cells. Nucleic Acids Res. 2020;48(W1):W509-W514. doi:10.1093/nar/gkaa407 
28. Li B, Severson E, Pignon JC, et al. Comprehensive analyses of tumor immunity: implications for cancer immunotherapy. Genome Biol. 2016;17(1):174. doi:10.1186/s13059-016-1028-7

29. Li T, Fan J, Wang B, et al. TIMER: a web server for comprehensive analysis of tumor-infiltrating immune cells. Cancer Res. 2017;77(21): e108-e110. doi:10.1158/0008-5472.CAN-17-0307

30. Newman AM, Liu CL, Green MR, et al. Robust enumeration of cell subsets from tissue expression profiles. Nat Methods. 2015;12 (5):453-457. doi:10.1038/nmeth.3337

31. Hall P, Ploner A, Bjohle J, et al. Hormone-replacement therapy influences gene expression profiles and is associated with breast-cancer prognosis: a cohort study. BMC Med. 2006;4:16. doi:10.1186/1741-7015-4-16

32. Pawitan Y, Bjohle J, Amler L, et al. Gene expression profiling spares early breast cancer patients from adjuvant therapy: derived and validated in two population-based cohorts. Breast Cancer Res. 2005;7(6): R953-964. doi:10.1186/bcr1325

33. Vasaikar SV, Straub P, Wang J, Zhang B. LinkedOmics: analyzing multi-omics data within and across 32 cancer types. Nucleic Acids Res. 2018;46(D1):D956-D963. doi:10.1093/nar/gkx1090

34. Bo C, Wu Q, Zhao H, Li X, Zhou Q. Thymosin alpha1 suppresses migration and invasion of PD-L1 high-expressing non-small-cell lung cancer cells via inhibition of STAT3-MMP2 signaling. Onco Targets Ther. 2018;11:7255-7270. doi:10.2147/OTT.S177943

35. Donehower LA, Soussi T, Korkut A, et al. Integrated analysis of TP53 gene and pathway alterations in the cancer genome atlas. Cell Rep. 2019;28(5):1370-1384 e1375. doi:10.1016/j.celrep.2019.07.001

36. Stanton SE, Disis ML. Clinical significance of tumor-infiltrating lymphocytes in breast cancer. J Immunother Cancer. 2016;4:59. doi:10.1186/s40425-016-0165-6

37. Azimi F, Scolyer RA, Rumcheva P, et al. Tumor-infiltrating lymphocyte grade is an independent predictor of sentinel lymph node status and survival in patients with cutaneous melanoma. $J$ clin oncol. 2012;30(21):2678-2683. doi:10.1200/JCO.2011.37.8539

38. Boschat AC, Minet N, Martin E, Barouki R, Latour S, Sanquer S. CTP synthetase activity assay by liquid chromatography tandem mass spectrometry in the multiple reaction monitoring mode. JMS. 2019;54 (11):885-893. doi:10.1002/jms.4442

39. Yang C, Liu X, Cheng T, et al. LYAR suppresses beta interferon induction by targeting phosphorylated interferon regulatory factor 3 . J Virol. 2019;93(21). doi:10.1128/JVI.00769-19.

40. Prabhu L, Hartley AV, Martin M, Warsame F, Sun E, Lu T. Role of post-translational modification of the $\mathrm{Y}$ box binding protein 1 in human cancers. Genes Dis. 2015;2(3):240-246. doi:10.1016/j. gendis.2015.05.001

41. Finak G, Bertos N, Pepin F, et al. Stromal gene expression predicts clinical outcome in breast cancer. Nat Med. 2008;14(5):518-527. doi: $10.1038 / \mathrm{nm} 1764$

42. Radvanyi L, Singh-Sandhu D, Gallichan S, et al. The gene associated with trichorhinophalangeal syndrome in humans is overexpressed in breast cancer. Proc Natl Acad Sci USA. 2005;102(31):11005-11010. doi:10.1073/pnas.0500904102
43. Curtis C, Shah SP, Chin SF, et al. The genomic and transcriptomic architecture of 2000 breast tumours reveals novel subgroups. Nature. 2012;486(7403):346-352. doi:10.1038/nature10983

44. Campbell TM, Castro MAA, de Oliveira KG, Ponder BAJ, Meyer KB. ERalpha binding by transcription factors NFIB and $Y B X 1$ enables FGFR2 signaling to modulate estrogen responsiveness in breast cancer. Cancer Res. 2018;78(2):410-421. doi:10.1158/00085472.CAN-17-1153

45. Fujii T, Kawahara A, Basaki Y, et al. Expression of HER2 and estrogen receptor alpha depends upon nuclear localization of Y-box binding protein-1 in human breast cancers. Cancer Res. 2008;68 (5):1504-1512. doi:10.1158/0008-5472.CAN-07-2362

46. Bansal T, Tanveer N, Singh UR, Sharma S, Kaur N. Y-Box binding protein 1 expression in breast cancer and its correlation with hormone receptors and other prognostic markers. J Lab Physicians. 2018;10 (4):420-425. doi:10.4103/JLP.JLP 5818

47. Ito T, Kamijo S, Izumi H, Kohno K, Amano J, Ito K. Alteration of Y-box binding protein-1 expression modifies the response to endocrine therapy in estrogen receptor-positive breast cancer. Breast Cancer Res Treat. 2012;133(1):145-159. doi:10.1007/s10549-0111731-8

48. Habibi G, Leung S, Law JH, et al. Redefining prognostic factors for breast cancer: YB-1 is a stronger predictor of relapse and disease-specific survival than estrogen receptor or HER-2 across all tumor subtypes. Breast Cancer Res. 2008;10(5):R86. doi:10.1186/ bcr2 156

49. Dong S, Nie H, Li D, et al. Molecular cloning and characterization of Y-box gene (Rpybx) from Manila clam and its expression analysis in different strains under low-temperature stress. Anim Genet. 2020;51 (3):430-438. doi:10.1111/age.12919

50. Lyabin DN, Eliseeva IA, Ovchinnikov LP. YB-1 protein: functions and regulation. Wiley Interdiscip Rev RNA. 2014;5(1):95-110. doi:10.1002/wrna.1200

51. Mittal S, Brown NJ, Holen I. The breast tumor microenvironment: role in cancer development, progression and response to therapy. Expert Rev Mol Diagn. 2018;18(3):227-243.

52. McKinney EF, Smith KG. T cell exhaustion and immune-mediated disease-the potential for therapeutic exhaustion. Curr Opin Immunol. 2016;43:74-80. doi:10.1016/j.coi.2016.09.005

53. Sica A, Schioppa T, Mantovani A, Allavena P. Tumour-associated macrophages are a distinct M2 polarised population promoting tumour progression: potential targets of anti-cancer therapy. Eur $J$ Cancer. 2006;42(6):717-727. doi:10.1016/j.ejca.2006.01.003

54. Hessman CL, Hildebrandt J, Shah A, et al. YB-1 interferes with TNFalpha-TNFR binding and modulates progranulin-mediated inhibition of TNFalpha signaling. Int J Mol Sci. 2020;21(19):7076. doi:10.3390/ijms21197076

55. Cao X, Zhu N, Li L, et al. Y-box binding protein 1 regulates ox-LDL mediated inflammatory responses and lipid uptake in macrophages. Free Radic Biol Med. 2019;141:10-20. doi:10.1016/j. freeradbiomed.2019.05.032

\section{Publish your work in this journal}

Cancer Management and Research is an international, peer-reviewed open access journal focusing on cancer research and the optimal use of preventative and integrated treatment interventions to achieve improved outcomes, enhanced survival and quality of life for the cancer patient.
The manuscript management system is completely online and includes a very quick and fair peer-review system, which is all easy to use. Visit http://www.dovepress.com/testimonials.php to read real quotes from published authors. 